\title{
Bacillus subtilis biofilm formation and social interactions
}

\author{
Arnaouteli, Sofia; Bamford, Natalie C.; Stanley-Wall, Nicola R.; Kovács, Ákos T.
}

Published in:

Nature Reviews. Microbiology

Link to article, DOI:

$10.1038 / \mathrm{s} 41579-021-00540-9$

Publication date:

2021

Document Version

Peer reviewed version

Link back to DTU Orbit

Citation (APA):

Arnaouteli, S., Bamford, N. C., Stanley-Wall, N. R., \& Kovács, Á. T. (2021). Bacillus subtilis biofilm formation and social interactions. Nature Reviews. Microbiology, 19, 600-614. https://doi.org/10.1038/s41579-021-00540-9

\section{General rights}

Copyright and moral rights for the publications made accessible in the public portal are retained by the authors and/or other copyright owners and it is a condition of accessing publications that users recognise and abide by the legal requirements associated with these rights.

- Users may download and print one copy of any publication from the public portal for the purpose of private study or research.

- You may not further distribute the material or use it for any profit-making activity or commercial gain

- You may freely distribute the URL identifying the publication in the public portal

If you believe that this document breaches copyright please contact us providing details, and we will remove access to the work immediately and investigate your claim. 


\section{Bacillus subtilis biofilm formation and social interactions}

Sofia Arnaouteli ${ }^{1, *}$, Natalie C. Bamford ${ }^{1, *}$, Nicola R. Stanley-Wall ${ }^{1 \dagger}$ \& Ákos T. Kovács ${ }^{2 \dagger}$

*These authors contributed equally

${ }^{1}$ Division of Molecular Microbiology, School of Life Sciences, University of Dundee, Dundee, UK

${ }^{2}$ Bacterial Interactions and Evolution Group, DTU Bioengineering, Technical University of Denmark, Kongens Lyngby, Denmark

†email: n.r.stanleywall@dundee.ac.uk; atkovacs@dtu.dk 


\begin{abstract}
Biofilm formation is a process in which microbial cells aggregate to form collectives that are embedded in a self-produced extracellular matrix. Bacillus subtilis is a Gram-positive bacterium that is used to dissect the mechanisms controlling matrix production and the subsequent transition from a motile planktonic cell state to a sessile biofilm state. The collective nature of life in a biofilm allows emergent properties to manifest, and $B$. subtilis biofilms are linked with novel industrial uses as well as probiotic and biocontrol processes. In this Review, we outline the molecular details of the biofilm matrix and the regulatory pathways and external factors that control its production. We explore the beneficial outcomes associated with biofilms. Finally, we highlight major advances in our understanding of concepts of microbial evolution and community behaviour that have resulted from studies of the innate heterogeneity of biofilms.
\end{abstract}




\section{[H1] Introduction}

Bacterial biofilms are a mode of collective living that confers emergent properties to the inhabitants of these communities ${ }^{1}$. A self-produced extracellular matrix that encapsulates the cells and facilitates their attachment to surfaces, among other functions, is a hallmark of biofilm formation ${ }^{2}$. The biofilm research field is fast moving due to the biological relevance of these multicellular consortia to an array of advantageous and detrimental effects in natural systems and human applications.

Bacillus subtilis is a soil-dwelling, non-pathogenic, Gram-positive bacterium that is commonly found in association with plants and their rhizosphere. B. subtilis is a highly tractable microorganism for which a broad suite of genetic and molecular tools is available that facilitate in vitro manipulation and study. Consequently, $B$. subtilis has been the major model organism for the study of Gram-positive bacteria for many decades. Furthermore, B. subtilis has been exploited for several industrial applications, including the production of hydrolytic enzymes, fermentation of food and, most recently, as a probiotic ${ }^{3}$.

The ability of $B$. subtilis to switch from a motile to a sessile state has been utilized to study biofilm formation. The undomesticated, ancestral isolate NCIB 3610 is widely studied to explore the three types of well-structured, three-dimensional biofilms that B. subtilis typically forms in vitro: a pellicle biofilm that develops at an air-liquid interface, a colony biofilm that develops at an air-solid interface and a submerged surface-attached biofilm ${ }^{4-6}$ (Figure 1). B. subtilis also forms biofilms on biotic surfaces, including fungal hyphae ${ }^{7}$ and roots $^{8}$ and leaves ${ }^{9}$ of plants. Furthermore, natural B. subtilis strains form pellicle biofilms on processed food products ${ }^{10}$.

The B. subtilis extracellular matrix contains an exopolysaccharide (EPS) that is synthesised by the products of the 15-gene epsABCDEFGHIJKLMNO (epsA-O) operon ${ }^{4}$, protein fibres of TasA encoded by the tapA-sipW-tasA operon (tapA operon) ${ }^{11}$ and BslA (encoded by $b s l A$ ), a hydrophobin-like protein that confers hydrophobicity to the community $^{12,13}$. A role for extracellular DNA (eDNA) ${ }^{14}$ and mineral deposits as components of the extracellular matrix has also been documented ${ }^{15}$. There is a strong correlation between the molecules that are needed for biofilm formation on biotic and abiotic surfaces, validating the utility of the laboratory model systems ${ }^{16}$. 
A substantial focus of many research groups has been to identify the regulatory mechanisms that underpin biofilm formation and characterize the properties of the extracellular matrix components of $B$. subtilis biofilms. Over the past 20 years it has become apparent that a $B$. subtilis biofilm formed by a single isogenic species is a remarkably heterogeneous community, making it ideal for the study of evolution within biofilm communities. Furthermore, B. subtilis exhibits emerging ecological properties that are dependent on biofilm formation, which affect social behaviours and interactions with hosts, resulting in a shift in the focus of research towards these as yet underappreciated aspects of B. subtilis physiology.

In this Review, we describe the recent advances in our knowledge of the principles governing biofilm formation, including transcription regulation and composition of the matrix. We explore biofilm formation within the context of microbial interactions and expand on interactions of the B. subtilis biofilm with hosts, such as plant roots and the intestinal tract. Finally, we highlight the progress that has been made in determining the evolutionary processes that occur in biofilm populations. By illuminating the versatile nature of $B$. subtilis biofilms, we extend the utility of $B$. subtilis biofilm formation beyond its usefulness as a laboratory model to a multifaceted and functional system with real-world applications in agriculture and human health.

\section{[H1] The B. subtilis biofilm matrix}

Great advances have been made in the characterization of $B$. subtilis biofilm matrix components, which include the secreted proteins TasA, TapA and BslA, as well as a mineral scaffold, eDNA and an exopolysaccharide. B. subtilis biofilms have been compared to colloidal hydrogels, with the matrix corresponding to the cross-linked gel ${ }^{17}$.

\section{[H2] Products of the tapA operon}

The tapA operon encodes three proteins that are involved in $B$. subtilis biofilm formation: TapA, SipW and TasA.

[H3] TapA. TapA is a 253-residue multidomain secreted protein containing a predicted 43amino-acid signal peptide. Tap A has been described as a TasA assembly and anchoring protein $^{18}$. Secreted TapA contains three distinct structural domains with varying levels of intrinsic disorder ${ }^{19,20}$ : an N-terminal domain (amino acids 44-75) that is necessary for pellicle and colony biofilm formation, a central stable domain (amino acids 75-191) that forms a $\beta$ sandwich fold (Protein Data Bank (PDB) identifier (ID) 6HQC and 6QAY) and a C-terminal domain (192-253) that is highly disordered in solution. As only residues 44-57 of TapA are 
essential for biofilm structure, the central stable domain, which is also the mostly highly conserved region of TapA, currently has no known function ${ }^{20}$. Co-expression of the wild-type tapA allele and a variant with mutations in residues 50-68 (overlapping with the essential region) slowed pellicle formation, although the underlying mechanism is unknown ${ }^{21}$.

In vivo, TapA localizes to foci on the cell surface that are proposed to anchor TasA fibres to the cell wall and accelerate TasA fibre formation ${ }^{18}$. However, TapA is not necessary for TasA fibre formation and does not affect fibre architecture in vitro but does enhance polymerization $^{21-23}$. Interestingly, TapA forms $\beta$-sheet rich aggregates in isolation and associates with fibril structures in vivo even when TasA is absent ${ }^{22}$. Whether these $\Delta$ tasA-fibres consist solely of TapA or whether TapA just has a propensity to associate with fibril structures remains to be clarified. Nonetheless, function of TapA in the matrix is closely tied to that of TasA. Consistent with this, strains lacking TapA have lower levels of matrix-localized TasA, and full function of TasA and TapA in the matrix requires their concomitant expression within the same cells ${ }^{18}$.

[H3] SipW. SipW is a type I signal peptidase that post-translationally modifies both TasA and TapA by cleaving their $\mathrm{N}$-terminal signal peptides at the extracellular surface ${ }^{24}$ (Figure 2). The cytoplasmic C-terminal domain of SipW additionally activates expression of the epsA-O and tapA operons in surface adherent biofilms ${ }^{25}$, adding a supplementary function to this protein that is essential for biofilm formation.

[H3] TasA. Originally proposed as an antibiotic factor ${ }^{26}$, TasA has various emerging roles, including functions in biofilm structure, sliding motility, signalling and plant colonization. TasA is a 261 amino acid protein with a 27-residue signal peptide ${ }^{27}$. TasA is found as fibres in the biofilm matrix and is required for the structural integrity of the biofilm ${ }^{11,23}$. TasA-fibres have been isolated directly from biofilms and also produced from recombinant protein. The source of the fibres influences the level of amyloid character that is detected ${ }^{11,22,28,29}$. TasA fibres isolated from $B$. subtilis pellicles show binding of thioflavin T, Congo red and the amyloid-specific antibody A11, which suggests that these isolated fibres have amyloid properties $^{11}$. Subsequent biophysical and recombinant protein approaches have revealed that both amyloid and non-amyloid TasA fibres exist ${ }^{22,28,29}$.

Recombinant TasA (which lacks the signal peptide and thus corresponds to the mature secreted protein) forms biologically active fibres that are non-amyloidogenic and have a similar secondary structure to those isolated from biofilms ${ }^{28}$. Unlike curli, the amyloid fibres that are produced by Escherichia coli and other enteric bacteria (reviewed elsewhere ${ }^{23}$ ), monomeric TasA in B. subtilis is globular in solution and adopts a jelly-roll-like fold with a flexible helix- 
rich region ${ }^{29}$. At low $\mathrm{pH}$, TasA forms dense gel-like networks that are comparable to colloidal aggregation $^{30}$. Transmission electron microscopy (TEM) imaging of TasA fibres consistently reveals repeating units along the protofilament (smallest diameter fibres) axis that are estimated to be $\sim 5 \mathrm{~nm}$ in length ${ }^{22,28}$, similar to the size of the TasA monomer. Like amyloid fibres, biologically active TasA fibres are resistant to proteases but, unlike $\beta$-amyloid fibres, TasA fibres are susceptible to the detergent sodium dodecyl sulfate, suggesting key differences in fibre structure. NMR analysis of TasA fibres, after addition to $\Delta$ tasA cultures, showed some changes in structure between the monomeric and fibre forms, including increased $\beta$-sheet character $^{29}$. By contrast, another study found that the CD spectra of the monomer structure were highly similar to those recombinant fibres and fibres purified from B. subtilis biofilms ${ }^{28}$. These studies also showed that the N-terminal region of processed TasA is important for polymerization, as addition of a single amino acid to the N-terminus blocks polymerization and locks TasA in a monomeric form. Thus, although the structure of TasA fibres is still unknown, the importance of their role in $B$. subtilis biofilms is well established.

TasA additionally affects and has a regulatory role in cell physiology ${ }^{9,31}$. The presence of TasA fibres within the biofilm matrix stimulates expression of motility genes and downregulates matrix expression in subpopulations of cells, and thus contributes to colony spreading on surfaces ${ }^{31}$. Deletion of tasA has other pleiotropic effects, including the down-regulation of genes related to sporulation and an increase in expression of matrix and antimicrobial secondary metabolite-related genes ${ }^{9}$. Moreover, TasA is associated with the detergent-resistant fraction of the cell membrane and influences membrane fluidity. However, most strikingly, tasA deletion leads to a decrease in B. subtilis viability in biofilm-inducing conditions. These functions have been proposed to be separate from TasAfibre forming and structural roles, as a strain producing a biofilm-inactive TasA variant retained wild-type physiological traits ${ }^{9}$. However, the level of the variant TasA produced by this strain was lower than in the wild type, opening the alternative prospect that the level of TasA needed varies with different processes. Looking beyond $B$. subtilis, TasA orthologues also have multiple roles. For example, in Bacillus cereus, the TasA orthologue CalY acts as a cell-associated adhesion factor during early biofilm formation prior to its release from the cell by the SipW homologue ${ }^{32}$. It is safe to say that despite the many studies conducted to date on TasA and its orthologues, many questions remain about its active structure, biological functions and roles in different strains and species.

\section{[H2] The biofilm surface layer protein BslA}


BslA has two genetically separable functions, being required for both biofilm hydrophobicity and biofilm architecture ${ }^{13,33}$. BslA seems to act synergistically with TasA fibres and exopolysaccharide (EPS) to generate structural complexity in the biofilm, as production of these matrix components is unaltered in mutants lacking BslA but colony biofilm structure is compromised $^{34}$. Whereas bslA is transcribed uniformly in the biofilm population, the protein primarily localizes to the biofilm periphery, forming a hydrophobic coating ${ }^{12,13}$. The crystal structure of BslA provides insight into this aspect of its function, as it reveals an immunoglobin G-like fold with a cap region that can exist in either a cap-in hydrophilic state or a cap-out hydrophobic state ${ }^{35}$ (Fig. 2d). The protein forms a 2D lattice in vitro, creating an elastic film at interfaces, which has been studied by biophysical techniques (Box 1$)^{35}$. It is conjectured that at the biofilm-air interface BslA transforms from cap-in to cap-out formation and this transition leads to hydrophobicity of the biofilm surface.

A C-terminal Cys-x-Cys (CxC) motif is required for BslA oligomerization in the oxygenrich environment of the biofilm surface, and the motif is essential for conferring hydrophobicity to the community, but is not required for the complex architecture of the biofilm ${ }^{33}$. The importance of the $\mathrm{CxC}$ motif for hydrophobicity was further demonstrated when YweA, a BslA paralogue lacking the $\mathrm{CxC}$ motif, was used in genetic complementation studies. Native YweA was unable to reinstate either hydrophobicity or structural complexity in a bslA-deficient strain, whereas a YweA variant containing the $\mathrm{CxC}$ motif fully restored hydrophobicity and partially restored the complex architecture ${ }^{33}$. Biofilm hydrophobicity and the corresponding presence of BslA (and its dimerized form) confers resistance of the resident bacteria to chemical attack ${ }^{33}$. To date, BslA has been studied primarily in NCIB 3610 and its relevance in the non-laboratory environment or other isolates is yet to be elucidated.

\section{[H2] Biofilm matrix carbohydrates}

EPS is the main biofilm carbohydrate and is synthesised by the 15 gene products of the eps $A-O$ operon ${ }^{4}$, which is transcribed in propagating waves at the edge of the expanding colony biofilm ${ }^{36}$. In addition to its signalling function, EPS is required for complex colony structure, pellicle formation and water retention ${ }^{25,37-41}$. The protein tyrosine kinase EpsB and EpsA are predicted to regulate EPS production ${ }^{42}$. The operon also encodes a sugar dehydratase (EpsC), multiple putative glycosyltransferases (EpsD, EpsE, EpsF, EpsH, EpsI, and EpsJ), a putative pyruvyl transferase (EpsO) and a predicted polysaccharide transport protein (EpsK) ${ }^{43,44,45}$ (Figure 2). Bioinformatic analysis predicts that EpsL is a sugar transferase that possibly transfers the first sugar unit onto a lipid carrier. EpsG is a predicted transmembrane protein of 
unknown function. The function of a few of these proteins have been studied in more detail. EpsE is a bifunctional protein that is involved in EPS synthesis and directly inhibits motility by acting as a clutch on the flagella rotor $^{44}$. EpsC has $\mathrm{NAD}^{+}$-dependent UDP- $N$ acetylglucosamine 4,6-dehydratase activity ${ }^{46}$. EpsN can produce UDP-2,6-dideoxy 2acetamido 4-amino glucose from of UDP-2,6-dideoxy 2-acetamido 4-keto glucose in a pyridoxal 5'-phosphate-dependent manner ${ }^{47}$. EpsM can transfer acetyl groups to UDP-2,4,6trideoxy-2-acetamido-4-amino glucose to produce $N, N^{\prime}$-diacetylbacillosamine. Based on sequence homology and the in vitro activities of EpsC, EpsM, and EpsN, it has been proposed that these proteins may have a role in the biosynthesis of $N, N^{\prime}$-diacetylbacillosamine, a modified monosaccharide that is produced by some bacteria ${ }^{48}$.

The composition of EPS and whether it contains $N, N^{\prime}$-diacetylbacillosamine is still unclear owing to conflicting findings from multiple studies. One analysis found that EPS contained glucose, $N$-acetylgalactosamine and galactose (with about 3:2:1 ratio) ${ }^{49}$. Further supporting this finding, the galactose metabolism pathway was found to be important for biofilm formation and regulation of this pathway was interrelated to regulation of the epsA-O operon $^{49}$. By contrast, another study concluded that EPS is predominantly composed of mannose $(88 \%)$ and glucose $(12 \%)^{50}$. Furthermore, B. subtilis EPS is cross-reactive with an antibody raised against poly- $\beta-1,6-N$-acetyl-D-glucosamine (PNAG), a common biofilm exopolysaccharide $^{45}$. Further investigation of the structure of EPS and monosaccharide components is necessary.

\section{[H2] Other matrix components}

eDNA is a common matrix component in bacterial biofilms and has been studied in diverse microbial systems ${ }^{51}$. The function of eDNA in $B$. subtilis biofilms is less explored than other biofilm systems but studies have found that eDNA is important for biofilm architecture and is required in the early stages of biofilm formation ${ }^{14}$. Treatment of $B$. subtilis biofilms with DNaseI during early biofilm development (12 h or earlier) led to an appreciable reduction in biofilm biomass but no difference was seen when the enzyme was added at later time points. Retention and localization of eDNA in the biofilm is also dependent on EPS production, a possible interaction that is further supported by isothermal titration calorimetry ${ }^{45}$.

Another highly anionic polymer of the $B$. subtilis matrix is poly- $\gamma$-glutamic acid ( $\gamma$ PGA), a linear polymer of L-glutamate and D-glutamate linked at the $\gamma$-carboxyl instead of at the $\alpha$-carboxyl that is typically used in proteins. The levels of $\gamma$-PGA in the matrix are highly 
dependent on the strain and growth conditions. Some strains, such as B. subtilis B-1, have been described as a $\gamma$-PGA dominant matrix former, whereas the common biofilm model strain NCIB 3610 produces very little $\gamma$-PGA ${ }^{52}$. Synthesis of $\gamma$-PGA is carried out by enzymes encoded by the pgsBCAE operon (previously known as ywsC-ywtABC): PgsB and PgsC are responsible for polymerization and PgsA and PgsE comprise the export machinery ${ }^{53}$ (Figure 2b). The export function of PgsE has been proposed based on findings from the highly homologous system of Bacillus anthracis. The presence of $\gamma$-PGA in the biofilm matrix is correlated with water retention and resistance to ethanol-induced dehydration ${ }^{54}$.

In addition to organic molecules, the biofilm matrix also contains inorganic compounds, such as metal ions and minerals. For example, B. subtilis induces calcite precipitation from calcium ions, which is correlated with increased colony biofilm wrinkling and complexity and may increase resistance to environmental assault by creating diffusion barriers $^{55,15}$. The conversion of calcium acetate to calcite crystals is linked to the increase in intra-colony $\mathrm{pH}$ during biofilm development, a process that is dependent on the ureA-C operon. The structure and morphology of calcium carbonate crystals are affected by TasA, TapA and EPS in vitro, suggesting that these molecules interact with calcium ions in the biofilm ${ }^{56}$.

Although the most abundant constituents of the biofilm matrix in $B$. subtilis have been identified, the interaction among them is largely unexplored. Intriguingly, substitution of specific conserved residues in TasA with a cysteine residue allows pellicle biofilm development in the absence of $\mathrm{EPS}^{57}$. However, this process is dependent on $\mathrm{BslA}^{57}$, suggesting that these TasA substitutions result in a novel inter-molecular interaction between these two components. Further investigation of matrix interactions and structures is required to fully understand this complex system.

\section{[H1] Regulation of biofilm formation}

Biofilm formation is an energetically expensive process that requires the transcription of a suite of genes to ensure timely production of the matrix molecules. Although the regulation of biofilm formation in B. subtilis has been extensively studied (reviewed elsewhere ${ }^{58,59}$ ), additional aspects of regulatory networks are still being discovered. Here, we briefly introduce the main components of these networks and review recent advances in the regulation of biofilm development mediated by intracellular circuitry.

\section{[H2] Intracellular regulatory pathways}


Biofilm formation and motility are described as mutually exclusive lifestyles, with cells in a bacterial population expressing genes that are necessary either for motility or for biofilm matrix production but not for both ${ }^{60}$. Consistent with this idea, B. subtilis exhibits heterogeneity in gene expression within the cell population (extensively reviewed elsewhere ${ }^{59}$ ), and transcription of the genes needed for motility is inversely correlated with that of the genes needed for biofilm matrix production. The transcription factor Spo0A is a critical regulator of B. subtilis biofilm formation ${ }^{4,5}$, with this regulatory pathway serving as an integration 'hub' for many competing and overlapping signals to control the proportion of cells in the biofilm that transcribe genes linked to biofilm matrix production. Initiation of biofilm formation is promoted when moderate levels of phosphorylated Spo0A (Spo0A P) are reached within a cell.

The sensor histidine kinases KinA, KinB, KinC and KinD are indirectly responsible for the phosphorylation of Spo0A via a phosphorelay, in which the phosphoryl groups are sequentially transferred from the kinases to the relay proteins Spo0F and Spo0B and finally to Spo0 $A^{61,62}$. The phosphorelay is affected by numerous Rap phosphatases and their corresponding Phr pentapeptides that together act as quorum-sensing systems ${ }^{63}$. These RapPhr systems affect biofilm development and evolution ${ }^{64}$, especially the plasmid-encoded RapP in strain NCIB $3610^{65}$. At threshold levels, Spo0A P directly represses transcription of $a b r B$, a transition state transcription repressor of several operons involved in biofilm formation, and concomitantly promotes the expression of $a b b A$, which encodes an anti-repressor of $\mathrm{AbrB}^{66}$.

A second Spo0A P-dependent anti-repressor pathway involves the transcription repressor SinR, a homotetramer that directly inhibits transcription of the matrix-encoding epsA-O and tapA operons ${ }^{60}$. Threshold levels of phospho-Spo0A trigger production of the SinR anti-repressor protein SinI and thereby stimulate transcription of the matrix-encoding operons. The first structures of full-length tetrameric SinR and dimeric SinI in solution shed light on how SinR binds to DNA and how SinI inhibits this function ${ }^{67}$. The formation of a flexible SinR homotetramer facilitates bending of the target DNA to enable repression of biofilm genes. SinI inhibits SinR from effectively binding DNA by forming SinR-SinI dimers that disrupt the formation of SinR homotetramers. SlrR is another antagonist of SinR which is induced by threshold levels of phosphor-Spo0 $\mathrm{A}^{68}$ and promotes transcription of the epsA-O and tapA operons, thus enabling the switch from a motile state to biofilm formation ${ }^{69}$. As levels of phospho-Spo0A P are not elevated homogeneously in the population, the regulatory network 
heterogeneously induces expression of matrix genes, creating subpopulations of matrix producer (ON-state) and non-producer (OFF-state) cells ${ }^{70-72}$.

Other transcription regulation systems also influence expression of biofilm-related genes by altering Spo0A P levels. For example, the phosphodiesterase YmdB influences the switch between biofilm and motility gene expression. YmdB deficiency has wide ranging effects on gene expression, resulting in reduced expression of biofilm genes and enhanced expression of motility genes, including hag (encoding flagellin) and autolysin genes ${ }^{73,74}$. By allowing the expression of SinR-repressed genes in a subpopulation of cells, YmdB acts as a regulatory protein that affects phenotypic heterogeneity in matrix gene expression ${ }^{75}$.

Another regulatory factor is the transcription termination factor Rho, an ATPdependent RNA helicase/translocase that represses genome-wide transcription ${ }^{76,77}$. Rho both directly and indirectly regulates motility, biofilm formation and sporulation ${ }^{78}$. In Rho-deficient cells, epsA-O and tapA operons are transcribed at low levels owing to an increase in SinRmediated repression of their promoters triggered by enhanced Spo0A phosphorylation.

The Spo0A mediated regulatory pathway is also modulated by the secondary messenger cyclic di-adenylate monophosphate (c-di-AMP). In addition to controlling growth, response to DNA damage and sporulation ${ }^{79,80}$, c-di-AMP also reduces expression of the tapA and epsA-O operons, leading to impaired biofilm formation ${ }^{81}$. While disruption of $\sin R$ restores biofilm formation under increased intracellular c-di-AMP concentrations, c-di-AMP accumulation does not affect the intracellular levels of SinR, suggesting that the nucleotide affects the activity of SinR. However, another study found that increased intracellular levels of c-di-AMP led to increased expression of the tapA operon. Furthermore, B. subtilis encodes two putative c-diAMP transporters that are involved in c-di-AMP secretion and facilitate root colonization. Thus, B. subtilis can sense and respond to extracellular c-di-AMP, uncovering a potential role of this second messenger in inter-bacterial communication ${ }^{82}$. Given the differences in experimental results, it is possible that the effect of intracellular c-di-AMP levels or the ability to secrete c-di-AMP changes in response to environmental conditions. c-di-AMP is not the only secondary messenger molecule that affects cell differentiation in B. subtilis. Cyclic diGMP (c-di-GMP) signalling directly inhibits motility in B. subtilis through the interaction of the putative c-di-GMP receptor YpfA with the flagellar motor protein MotA ${ }^{83}$. However, the effect of c-di-GMP levels on biofilm formation is disputed ${ }^{83,84}$.

\section{[H2] The Y-complex}


The Y-complex comprises three proteins, RicA, RicF and RicT (also known as YmcA, YlbF and YaaT, respectively), which contribute to B. subtilis biofilm formation. Inactivation of these proteins results in impaired biofilm-forming ability ${ }^{38,60}$, although the mechanism underpinning this phenotype remains unclear. A study reported that the Y-complex regulates biofilm formation by accelerating the phosphorylation of $\mathrm{SpoOA}^{85}$, whereas another found that SinR mRNA and protein levels are increased in Y-complex mutants and demonstrated that the Y-complex interacts with and controls the activity of RNaseY, which destabilizes $\sin R$ mRNA $^{86}$. However, more recent findings dispute the mRNA destabilization model and suggest that in addition to accelerating Spo0A phosphorylation, the Y-complex induces biofilm formation through an unknown Spo0A-independent pathway ${ }^{87,88}$.

\section{[H2] Sensing stress}

Stress conditions influence biofilm formation in diverse bacteria ${ }^{89}$. In B. subtilis, the alternative sigma factor sigma $\mathrm{B}\left(\sigma^{\mathrm{B}}\right)$ is activated by stressors, including heat, salt and starvation conditions ${ }^{90}$. Activity of $\sigma^{\mathrm{B}}$ manifests as a series of stochastic pulses within cells in the biofilm, showing maximal expression at the upper surfaces of the community ${ }^{91}$. Stochastic pulsing of $\sigma^{\mathrm{B}}$ appears to provide an adaptation mechanism for cells to either activate $\sigma^{\mathrm{B}}$ or sporulate, thus allowing cells in these different states to coexist in the same zone of the biofilm structure. Heterogeneous expression of biofilm components is linked with changes in biofilm structure and robustness over both space and time ${ }^{71}$. For example, during pellicle formation a substantial increase in biomass robustness is correlated with an increase in eps $A-O$ expression, and with tasA expression being almost universally activated in the cells of the biofilm population $^{71}$. These data also reveal heterogeneity between the transcription profiles from the tapA and epsA-O promoters. The genetically homogeneous population in the pellicle undergoes a phenotypic segregation into three phenotypically distinct subgroups producing different levels of EPS and TasA: matrix non-producers, EPS producers, and generalist cells that produce both major components. This differentiation of the population can be viewed as a 'phenotypic division of labour' where specialization leads to maximum population efficiency ${ }^{92}$.

The repertoire of regulatory network components influencing biofilm development in $B$. subtilis is extensive. However, we still do not fully understand whether these pathways are active under all conditions or only become active in specific environmental conditions. Heterogeneous activation of transcriptional regulators further increases the phenotypic diversity of $B$. subtilis cells within the biofilms and enhances survival. 


\section{[H1] Impact of microbial interactions}

The drive to understand microbial interactions and re-create simplified, ecologically relevant habitats has led to research on mixed biofilms. In the rhizosphere, B. subtilis cohabitates with many soil-dwelling bacteria and fungi, and interactions between these organisms and B. subtilis can modulate biofilm structure and robustness (comprehensively reviewed elsewhere ${ }^{93}$ ).

\section{[H2] Biofilm modulation by other species}

Numerous bacterial species modulate $B$. subtilis biofilm formation and structure ${ }^{94-96}$. The expression of $B$. subtilis biofilm genes is induced during co-culture with other members of the Bacillus genus ${ }^{97}$. B. cereus produces and secretes thiazolyl peptide antibiotics termed thiocillins, which trigger matrix production in B. subtilis ${ }^{98}$. Interestingly, abolishing the antibiotic activity of thiocillin did not affect its ability to induce biofilm gene expression, suggesting that secondary metabolites can have an alternative function in addition to antibiosis. By contrast, Pseudomonas putida and Pseudomonas protegens hinder biofilm-specific gene expression when co-cultured with $B$. subtilis, a behaviour that is mediated by secretion of the antimicrobial 2,4-diacetylphloroglucinol (DAPG) that delays tapA gene expression at subinhibitory concentrations ${ }^{99}$. DAPG alters $B$. subtilis biofilm growth and phenotypic differentiation adjacent to $P$. protegens. While various environmental signals are sensed by histidine kinases in $B$. subtilis that affect Spo0A P levels, development of $B$. subtilis biofilms can also be affected in a Spo0A-independent manner ${ }^{95}$. The soil bacterium LysiniBacillus fusiformis induces wrinkle formation in B. subtilis colonies through a diffusible primary metabolite, hypoxanthine ${ }^{95}$. The induction of biofilm wrinkle formation was hypothesized to be mediated by localized $B$. subtilis cell death caused by increased intracellular hypoxanthine levels, as deletion of hypoxanthine transporters in $B$. subtilis abolished induction of wrinkles by $L$. fusiformis ${ }^{95}$.

Mixed cross-kingdom biofilms are also observed. For example, B. subtilis colonization of the hyphae of both the filamentous black mould fungus Aspergillus niger and hyphae of the basidiomycete mushroom Agaricus bisporus depends on TasA and EPS ${ }^{7}$. B. subtilis also forms biofilms on the ectomycorrhizal fungus Laccaria bicolor, where eDNA has been suggested to contribute to this interaction ${ }^{100}$. The relationship can also be bidirectional; the plant pathogen Fusarium culmorum induces biofilm formation in B. subtilis through decreased expression of $\sin R$ and therefore enhanced tas $A$ transcription ${ }^{101}$. In addition, the presence of fungal plant 
pathogens can also induce the expression of fungitoxic secondary metabolites by $B$. subtilis to increase its competitive edge $\mathrm{e}^{102,103}$.

\section{[H2] Synergism in multispecies biofilms}

Dual-species biofilms can gain unique characteristics as compared to their respective monoculture colonies ${ }^{96}$. The soil dwelling and rhizospheric bacterium, Pantoea agglomerans has increased antibiotic resistance when it cohabitates with $B$. subtilis ${ }^{94}$, which requires $B$. subtilis TasA and a P. agglomerans exopolysaccharide. Similarly, B. subtilis adherence to a Streptococcus mutans biofilm also depends on Tas $\mathrm{A}^{96}$. Interestingly, production of B. subtilis biofilm components is induced by $S$. mutans, suggestive of a feedback loop. In addition to synergistic mixed-species communities, the $B$. subtilis matrix has a protective role in encounters with other microbes. On the leaf surface, Pseudomonas chlororaphis invades the B. subtilis population when the biofilm matrix is absent, while the type VI secretion system of P. chlororaphis additionally stimulates sporulation of $B$. subtilis ${ }^{104}$. In the presence of the biofilm matrix, B. subtilis co-exists with $P$. chlororaphis, forming a community that collectively promotes plant growth and protects from pathogens. The B. subtilis matrix is also needed to create a spore-filled biofilm megastructure upon interaction with Myхососcus xanthus $^{105}$. The emergent properties of the $B$. subtilis biofilm can also affect $B$. subtilis and other microbes in the surrounding environment. Potassium ion channel-mediated electrical signals generated by a $B$. subtilis biofilm alter the membrane potential of distant cells and modulate their motility ${ }^{106}$. This communication mechanism extends beyond the cells within the B. subtilis population; for example, Pseudomonas aeruginosa cells are attracted to the electrical signal released by the $B$. subtilis biofilm ${ }^{107}$.

\section{[H2] Interspecific competition}

Engagement with a microbial neighbour can also be less amicable, as $B$. subtilis expresses toxins and other effectors to dominate a niche. The build-up of the extracellular ironchelating molecule pulcherriminic acid in the region surrounding colony biofilms initiates growth arrest of the producing community while concomitantly preventing invasion by neighbouring microorganisms ${ }^{108,109}$. Other bioactive compounds, including surfactins, the cannibalism toxin SDP (cannibalism in this context refers to inducing lysis of conspecific cells and cells of other species to release nutrients for biofilm growth), sporulation killing factor and various other secondary metabolites, are of interest owing to their potential agricultural use as biocontrol agents. For example, B. subtilis biofilms outcompete Serratia plymuthica both in 
vitro and on plant roots by producing bacillaene, a non-ribosomally synthesized antibiotic ${ }^{110}$. B. subtilis biofilms on plant roots also form a zone of exclusion that prevents E. coli colonization, by an unidentified mechanism ${ }^{111}$. Competition is also present between Bacillus spp., as B. subtilis biofilms expand and subsume neighbouring Bacillus simplex colonies, by secreting SDP and the lipopeptide toxin surfactin, which induce B. simplex cell death ${ }^{112}$. Of note, $B$. subtilis produces a biofilm-specific antibacterial toxin, YIT, which contributes to its competitiveness against sensitive strains or species ${ }^{113}$. YIT is produced during biofilm formation from an operon paralogous to that of SDP but unlike SDP can permeate the biofilm matrix. Depending on their concentration, many of these compounds can also affect 'kin' (or conspecific) strains, leading to nuance in interspecific and intraspecific microbial interactions ${ }^{114-116}$. Imaging mass spectrometry provides a powerful novel approach to study how specific metabolites and peptides are spatially distributed in the above-mentioned interactions and will contribute to understanding of how interspecific and intraspecific interactions modulate $B$. subtilis biofilm development.

\section{[H1] Biofilm formation on plant roots}

B. subtilis is a well-known biocontrol agent that is widely used in agriculture ${ }^{117}$. However, the underlying mechanism of these plant-microbe interactions and how they contribute to preventing pathogenic microorganism colonization has only recently been explored $^{118}$. Advances in model systems and experimental designs have allowed visualization of $B$. subtilis biofilm dynamics in the rhizosphere over longer time scales. Although these studies primarily examined Arabidopsis thaliana, other plant species are beginning to be used $^{8,111}$ ( BOX 2). A large diversity of $B$. subtilis strains have been isolated from the rhizosphere ${ }^{119,120} 16$. For example, 50 unique isolates were collected from the rhizosphere of cocoa trees (Theobroma cacao), of which $90 \%$ were able to form robust biofilms ${ }^{119}$. Similarly, another study found variation in the ability of rhizosphere $B$. subtilis isolates to form biofilms in vitro and on tomato plant roots ${ }^{16}$. The variation among $B$. subtilis strains is not only observed in their ability to form biofilms but also in additional plant growth-promoting and biocontrol traits, such as mineral solubilization, indole acetic acid production, siderophore secretion and surfactin production ${ }^{16} 120$.

\section{[H2] Colonization of plant roots}

Before biofilms can form, motile cells need to find and adhere to the root surfaces. Indeed, both motility and chemotaxis are required for $B$. subtilis colonization of $A$. thaliana 
roots ${ }^{121}$ Of the 10 known chemoreceptors encoded in the $B$. subtilis genome, McpB, McpC and TlpC mediate attraction to $A$. thaliana root exudates ${ }^{121}$ (Figure 3). Interestingly, colonization of growing roots is not solely dependent on these chemoreceptors, suggesting that redundancies are present in the chemotaxis and other sensing systems that respond to root-derived signals ${ }^{121}$. B. subtilis preferentially colonizes the root differentiation and elongation zone, as shown for $A$. thaliana and cotton plants ${ }^{111,122}$. Microfluidic imaging techniques showed that accumulation of $B$. subtilis at the elongation zone began within 20 min of inoculation ${ }^{111}$. Intercellular signalling between $B$. subtilis cells (that is, secretion of c-di-AMP) is important for successful attachment to the root, as the c-di-AMP permeases $\mathrm{YcnB}$ and YhcA are necessary for efficient colonization of $A$. thaliana roots $^{82}$.

B. subtilis matrix components are required for colonization and biofilm formation on plant roots ${ }^{16,123,124}$. Deletion of the eps $A-O$ operon or tas $A$ coding region result in defective biofilm formation on tomato seedling roots ${ }^{16,124}$. Interestingly matrix-deficient $B$. subtilis mutants only establish dense biofilms on $A$. thaliana roots when co-inoculated with wild-type cells, suggesting that matrix components are shared ${ }^{92,123}$. Overproduction of $\gamma$-PGA also contributes to root colonization and persistence of some $B$. subtilis strains, although it is not essential $^{125,126,52}$. The role of surfactins in B. subtilis biofilm formation on root surfaces is unclear $^{120,127,128}$. Although surfactin production alters the structure of colony and pellicle biofilms in vitro, the most recent data suggest that surfactin does not affect biofilm formation on plant roots ${ }^{120,128}$.

\section{[H2] Ecological interactions}

Our molecular understanding of plant signalling and interactions with B. subtilis continues to advance. Plant polysaccharides, including xylan, pectin, and arabinogalactan, have been found to have a crucial role in stimulating $B$. subtilis biofilm formation ${ }^{123,129}$. These molecules perform this function in two distinct ways; first, they induce biofilm matrix gene expression by stimulating the activity of histidine kinases that phosphorylate the master regulator Spo0A and, second, they are processed and incorporated into the biofilm matrix ${ }^{123}$. Digestion and utilization of galactose might occur through the galactan utilization pathway, as B. subtilis binding to plant roots or $\beta$-1,4-galactobiose leads to derepression of the galactan utilization (gan) operon ${ }^{129}$.

Root-associated biofilms also represent an environment for kin discrimination (differential interaction with organisms based on relatedness) among $B$. subtilis strains or isolates. The ability of $B$. subtilis strains to create mixed-isolate biofilms on plant roots directly 
correlates with the phylogenetic distance of the strains, suggesting that there is antagonism between non-kin strains during rhizoplane colonization of the rhizoplane (the region of the rhizosphere in which the root is in contact with the soil) ${ }^{114}$. Microbes that are potentially valuable to plants must compete with other organisms in the ecosystem to occupy this space. Tomato plant exudates induce bacillaene production by $B$. subtilis during colonization, which leads to increased killing efficacy of the rhizoplane competitor S. plymuthica ${ }^{110}$. In addition, superior B. subtilis colonization compared with S. plymuthica also mediated increased plant systemic resistance against the plant pathogen Pseudomonas syringae ${ }^{110}$. This interplay suggests that a complex symbiosis exists between $B$. subtilis and the plant host, which is driven by evolutionary adaptation.

\section{[H1] Biofilms in the intestinal tract}

Commensal bacteria are pivotal to human health, as they prompt the host's immune system to induce protective responses that prevent colonization and invasion by pathogenic species. Interest is growing in the identification of the genes and mechanisms that are utilized by beneficial microbes to limit disease caused by invading pathogens. Although $B$. subtilis strains are mainly found in the soil, they have also been isolated from human skin and the gastrointestinal tract, showing that the bacterium has adapted its physiology to survive under diverse conditions ${ }^{130,131}$. B. subtilis is one of the predominant microorganisms used in probiotic products, despite the underlying mechanisms by which the bacterium can restore and maintain a healthy gut flora being largely unknown. Studies have begun to link the protective effect of B. subtilis to its biofilm production (Figure 4).

In a mouse model, wild-type B. subtilis confers protection from Citrobacter rodentium, an enteric pathogen that causes acute colitis, unlike an EPS-deficient B. subtilis strain ${ }^{132}$. Protection by B. subtilis EPS is a result of host immune modulation rather than prevention of pathogen colonization or disruption of the epithelium. EPS prevents colitis in a TLR4dependent manner that requires myeloid cells ${ }^{50}$. EPS induces the formation of antiinflammatory M2 macrophages that produce TGF $\beta$ and PDL1 to broadly inhibit the activation of T cell responses ${ }^{133}$. Furthermore, B. subtilis EPS also protects against Staphylococcus aureus systemic infection. Macrophages from EPS-treated mice exhibited an M2 phenotype and also restricted growth of internalized $S$. aureus through the production of reactive oxygen species ${ }^{134}$. Altogether, these data suggest that $B$. subtilis EPS can induce the differentiation of immune 
cells that have antibacterial and anti-inflammatory properties, which ultimately contribute to increased host survival.

Caenorhabditis elegans is a nematode that is widely used to study bacterial pathogenicity and host immunity. B. subtilis was first found to be beneficial to C. elegans, as substituting E. coli OP50 for $B$. subtilis as a food source extended the longevity of the $C$. elegans daf-2 and age-1 long-lived mutants ${ }^{135}$. Biofilm formation by B. subtilis was later identified as one of the factors that prolong C. elegans longevity. Two molecules produced by B. subtilis that extend $C$. elegans lifespan are the quorum-sensing molecule CSF and nitric oxide. Synthesis of both molecules is enhanced in B. subtilis biofilm-forming conditions, compared with planktonic growth ${ }^{136}$. B. subtilis biofilm formation in C. elegans also confers resistance against different types of stress, such as heat and oxidative stress ${ }^{137}$. The protective role of the $B$. subtilis biofilm was additionally demonstrated against the causative agents of two detrimental neurodegenerative diseases, $\alpha$-synuclein in Parkinson disease and amyloid- $\beta$ in Alzheimer disease. A probiotic B. subtilis strain inhibits aggregation of $\alpha$-synuclein and cleared $\alpha$-synuclein aggregates in a $C$. elegans synucleinopathy model. Biofilm-deficient strains exhibited higher rates of $\alpha$-synuclein aggregation than in a wild-type biofilm-proficient strain $^{138}$. Similarly, B. subtilis biofilm formation in the gut contributes to the role of $B$. subtilis in protecting against amyloid- $\beta$-related toxicity in a $C$. elegans Alzheimer disease model ${ }^{139}$. Research is currently ongoing to further investigate the potential of $B$. subtilis to treat or prevent neurodegenerative diseases. Future developments in the field, such as imaging technologies, chemical profiling, and using genetically modified host systems will create opportunities to identify the underlying mechanisms involved in host-microbe interaction during $B$. subtilis biofilm formation.

\section{[H1] Experimental evolution in the biofilm}

An emerging topic in biofilm research is mapping the principles that govern evolution in biofilm populations. Studies have uncovered novel insights into genetic and phenotypic diversity and interactions between matrix elements that would otherwise have remained undetected. Intriguingly, repeated cultivation of B. subtilis in shaking planktonic conditions results in rapid genetic differentiation, discernible as derivative isolates with distinct colony morphotypes $^{140}$. The variability in colony biofilm structures was connected to mutation of $\sin R$ and the resultant alteration in expression of biofilm-related genes. Similarly, pellicle biofilms formed by $B$. subtilis can be exploited for studying experimental evolution of B. subtilis 
biofilms (Figure 5), primarily because of the degree of heterogeneity observed within the otherwise robust biofilm population. $B$. subtilis can diversify into distinct colony variants that exhibit differences in their biofilm formation abilities and expression of biofilm-related genes $^{141}$. The evolved colony morphotypes also display differences in surface complexity and hydrophobicity. Interestingly, the co-cultivation of derived morphotypes increases the population yield (that is, the abundance of cells) compared with the ancestral strain NCIB $3610^{141}$. The increased biofilm dimensions with a mixed population are driven by the shareability of the secreted matrix components ${ }^{71,92}$. Indeed, a mixture of tas $A$ and eps $A-O$ mutant derivative strains rescue pellicle biofilm development by 'public goods' sharing ${ }^{92}$. Intriguingly, a mixture of two genetic derivative strains, one lacking EPS and the other TasA, enhances pellicle productivity (the number of cells residing in the biofilm) and plant root colonization compared with that of a strain that produces both components, revealing a 'genetic division of labour' ${ }^{92}$. Similarly, both EPS-containing supernatant and purified TasA protein fibres rescue biofilm formation by the respective mutants ${ }^{11,18,22,23,92}$. However, the degree of sharing of the two matrix components differs and TasA cannot be shared as widely in the population as EPS ${ }^{71}$. Such an imbalance creates a collapse of genetic division of labour when the mixture of eps and tas $A$ mutants is evolved for a few hundred generations ${ }^{57}$. Eventually, tasA and eps mutants lacking one of the matrix components enhances the air-medium interface colonization ability, either by enhancing production of EPS or by altering TasA protein properties, respectively. In the EPS-deficient strain, introduction of cysteine residues at specific residues in TasA generates thicker fibres than those produced by the wild-type ancestor ${ }^{57}$. However, in the presence of EPS, strains with cysteine-substituted TasA are at a disadvantage, as hydrophobicity is hampered, possibly due to disulphide bond formation between the newly substituted cysteine in TasA and the cysteine residues of BslA ${ }^{33,141}$. Importantly, experimental evolution studies using strains that lack robust biofilm formation ability have helped to uncover novel features of TasA.

The rapid evolution of mutants with altered biofilm formation kinetics also highlights adaptation pathways in B. subtilis. A strain deficient in the phosphodiesterase YmdB does not form biofilms, although the ability to form pellicles in biofilm-inducing medium is rapidly recovered due to acquisition of mutations in $\sin R$. Similarly, mutants lacking functional propelling flagella in $B$. subtilis rapidly attain mutations in $\sin R$ and increased expression of biofilm genes, an adaptation that increases competitiveness compared with its ancestor ${ }^{142,143}$. Of note, the range of mutations in $\sin R$ overlap in $y m d B$ and motility-deficient hag background, highlighting a general evolutionary adaption in strains lacking biofilm-gene expression. 
Experimental evolution can also be exploited to investigate how matrix-deficient derivative strains affect biofilm-proficient wild-type strains. Strains deficient in both EPS and TasA are unable to exploit the matrix nor do they incorporate into the pellicles formed by the wild-type strain ${ }^{144}$. However, after de novo evolved competition mediated by recombination of cryptic phi3T phage with the SP $\beta$ prophage region of $B$. subtilis, the double-mutant strain partially increases in the biofilms ${ }^{144,145}$. However, it remains unclear why the matrix-deficient double-mutant strain benefits more from this competition than the wild-type strain. By contrast, eps $A-O$ or tasA single-mutant strains can readily exploit the matrix and benefit from this 'public good', as the cells disengage from the metabolic burden of producing the respective matrix component and therefore act as 'cheaters' 146 .

The spatial structure of biofilms is a parameter that can affect bacterial adaptation. The ability of $B$. subtilis to form biofilms has been exploited to study metabolic adaptation in comparison with planktonic growth or other differentiation processes, such as sporulation ${ }^{147}$. It will be interesting to uncover distinct bacterial evolutionary and adaptation processes during biofilm development. Ongoing research is currently being performed to expand our understanding of the evolution of $B$. subtilis biofilms directly on the plant root, connecting experimental evolution with plant host-associated biofilms.

\section{[H1] Applications of biofilms}

B. subtilis is recognized as a superb model system to study the matrix components, gene regulation and social interactions during biofilm development. Systematic understanding of how B. subtilis biofilms can be harnessed for use in probiotics has only recently been explored. Intriguingly, humans have a long history of exploiting microorganisms and the use of Bacillus spp. can be traced back to the production of traditional fermented foods, including the Japanese soybean breakfast food natto and the Chinese delicacy pidan (Century egg). Today, a diverse range of processes exploit Bacillus spp. across many sectors of the economy, and more recently several of the innovations are both informed and inspired by knowledge of the processes and materials that underpin biofilm formation. The secreted matrix molecules have unique features that lend themselves to novel biotechnological or biomedical purposes. BslA undergoes a limited structural metamorphosis at an interface such that a surface-active region becomes exposed $^{35}$, which enables BslA to stabilize a variety of multiphase formulations ${ }^{148}$. Surfaceactive proteins have widespread uses in both nature and bioengineering, thus recombinant BslA has been proposed as a tool for stabilization of ice cream to slow down the melting process ${ }^{149}$. 
Novel industrial uses that require the direct use of $B$. subtilis biofilms have also emerged over the last decade, including the proposed use of B. subtilis biofilms for calcite precipitation ${ }^{150}$. Looking beyond the use of $B$. subtilis as a probiotic or biological control agent, B. subtilis biofilms can be repurposed as a protective coating for other probiotic bacteria in the gut ${ }^{151}$. Building on the viscoelastic properties of the whole biofilm, B. subtilis biofilms have been postulated as 'living materials' that can be used for a variety of purposes ${ }^{152}$.

\section{[H1] Conclusions and future perspectives}

The future of $B$. subtilis research will undoubtedly be driven by next-generation experimental approaches, ecological relevance and applications triggered by a basic understanding of the molecular components of biofilm formation. Recent developments in single-cell transcriptome analysis should help to reveal heterogeneity in global gene expression profiles within both single- and mixed-species biofilms, while progress in imaging mass spectrometry at high spatial resolution will allow understanding of the chemical language that influences biofilm gene expression heterogeneity within microbial communities. Furthermore, increased resolution and sensitivity of imaging approaches applied to analyse biofilms of $B$. subtilis and co-inhabiting microbes directly within the host at continuous time scales can be applied to investigations of diverse environments ranging from the plant rhizosphere to the animal digestive system. While we have learnt a lot from dissecting $B$. subtilis biofilm formation in the laboratory, the discovery of B. subtilis biofilms in situ in nature still requires further technological and conceptual approaches. The first imaging of a naturally formed $B$. subtilis biofilm in situ in the soil is still awaited. 


\section{References}

1 Flemming, H. C. \& Wuertz, S. Bacteria and archaea on Earth and their abundance in biofilms. Nat Rev Microbiol 17, 247-260, doi:10.1038/s41579-019-0158-9 (2019).

2 Flemming, H. C. \& Wingender, J. The biofilm matrix. Nat Rev Microbiol 8, 623-633, doi:nrmicro2415 [pii]10.1038/nrmicro2415 (2010).

3 Marzorati, M. et al. Bacillus subtilis HU58 and Bacillus coagulans SC208 Probiotics Reduced the Effects of Antibiotic-Induced Gut Microbiome Dysbiosis in An M-SHIME((R)) Model. Microorganisms 8, doi:10.3390/microorganisms8071028 (2020).

4 Branda, S. S., Gonzalez-Pastor, J. E., Ben-Yehuda, S., Losick, R. \& Kolter, R. Fruiting body formation by Bacillus subtilis. Proc Natl Acad Sci U S A 98, 11621-11626 (2001).

This is the first paper demonstrating the complex architecture manifested in the $B$. subtilis NCIB360 biofilm.

5 Hamon, M. A. \& Lazazzera, B. A. The sporulation transcription factor Spo0A is required for biofilm development in Bacillus subtilis. Mol Microbiol 42, 1199-1209 (2001).

6 Sanchez-Vizuete, P. et al. Identification of ypqP as a New Bacillus subtilis Biofilm Determinant That Mediates the Protection of Staphylococcus aureus against Antimicrobial Agents in Mixed-Species Communities. Appl Environ Microb 81, 109-118, doi:10.1128/Aem.02473-14 (2015).

$7 \quad$ Kjeldgaard, B. et al. Fungal hyphae colonization by Bacillus subtilis relies on biofilm matrix components. Biofilm 1, 100007 (2019).

This is the first study showing the role of the matrix components TasA and EPS in B. subtilis biofilm formation on fungal hyphae.

8 Noirot-Gros, M. F. et al. Functional Imaging of Microbial Interactions With Tree Roots Using a Microfluidics Setup. Front Plant Sci 11, 408, doi:10.3389/fpls.2020.00408 (2020).

9 Camara-Almiron, J. et al. Dual functionality of the amyloid protein TasA in Bacillus physiology and fitness on the phylloplane. Nat Commun 11, 1859, doi:10.1038/s41467-020-15758-z (2020).

10 Kovacs, A. T. \& Dragos, A. Evolved Biofilm: Review on the Experimental Evolution Studies of Bacillus subtilis Pellicles. J Mol Biol 431, 4749-4759, doi:10.1016/j.jmb.2019.02.005 (2019).

11 Romero, D., Aguilar, C., Losick, R. \& Kolter, R. Amyloid fibers provide structural integrity to Bacillus subtilis biofilms. Proc Natl Acad Sci U S A 107, 2230-2234, doi:0910560107 [pii]

10.1073/pnas.0910560107 (2010).

This is the first study to identify TasA as a fibre-forming protein that is integral to biofilm formation.

12 Hobley, L. et al. BsIA is a self-assembling bacterial hydrophobin that coats the Bacillus subtilis biofilm. P Natl Acad Sci USA 110, 13600-13605, doi:10.1073/pnas.1306390110 (2013).

This paper reported the first crystal structure of BsIA and demonstrated the hydrophobin-like ability of this unique protein by combining in vivo and in vitro biophysical techniques.

13 Kobayashi, K. \& Iwano, M. BsIA (YuaB) forms a hydrophobic layer on the surface of Bacillus subtilis biofilms. Mol Microbiol 85, 51-66, doi:10.1111/j.1365-2958.2012.08094.x (2012).

14 Peng, N. et al. The exopolysaccharide-eDNA interaction modulates 3D architecture of Bacillus subtilis biofilm. BMC Microbiol 20, 115, doi:10.1186/s12866-020-01789-5 (2020).

15 Keren-Paz, A., Brumfeld, V., Oppenheimer-Shaanan, Y. \& Kolodkin-Gal, I. Micro-CT X-ray imaging exposes structured diffusion barriers within biofilms. NPJ Biofilms Microbiomes 4, 8, doi:10.1038/s41522-018-0051-8 (2018).

16 Chen, Y. et al. Biocontrol of tomato wilt disease by Bacillus subtilis isolates from natural environments depends on conserved genes mediating biofilm formation. Environ Microbiol 15, 848-864, doi:10.1111/j.1462-2920.2012.02860.x (2013). 

and water binding in biofilms. Soft Matter 16, 6180-6190, doi:10.1039/d0sm00581a (2020). Romero, D., Vlamakis, H., Losick, R. \& Kolter, R. An accessory protein required for anchoring and assembly of amyloid fibres in B. subtilis biofilms. Molecular Microbiology, doi:10.1111/j.1365-2958.2011.07653.x (2011).

19 Abbasi, R. et al. The Bacterial Extracellular Matrix Protein TapA Is a Two-Domain Partially Disordered Protein. Chembiochem 20, 355-359, doi:10.1002/cbic.201800634 (2019).

20 Earl, C. et al. The majority of the matrix protein TapA is dispensable for Bacillus subtilis colony biofilm architecture. Mol Microbiol, doi:10.1111/mmi.14559 (2020).

21 Romero, D., Vlamakis, H., Losick, R. \& Kolter, R. Functional analysis of the accessory protein TapA in Bacillus subtilis amyloid fiber assembly. J Bacteriol 196, 1505-1513, doi:10.1128/JB.01363-13 (2014).

22 El Mammeri, N. et al. Molecular architecture of bacterial amyloids in Bacillus biofilms. Faseb J 33, 12146-12163, doi:10.1096/fj.201900831R (2019).

23 Erskine, E., MacPhee, C. E. \& Stanley-Wall, N. R. Functional Amyloid and Other Protein Fibers in the Biofilm Matrix. J Mol Biol 430, 3642-3656, doi:10.1016/j.jmb.2018.07.026 (2018).

24 Serrano, M. et al. A Bacillus subtilis secreted protein with a role in endospore coat assembly and function. Journal of Bacteriology 181, 3632-3643 (1999).

25 Terra, R., Stanley-Wall, N. R., Cao, G. \& Lazazzera, B. A. Identification of Bacillus subtilis SipW as a bifunctional signal peptidase that controls surface-adhered biofilm formation. Journal of Bacteriology 194, 2781-2790, doi:10.1128/JB.06780-11 (2012).

26 Stover, A. G. \& Driks, A. Secretion. localization and antibacterial activity of tasA, a Bacillus subtilis spore-associated protein. J. bacteriol. 181, 1664-1672 (1999).

27 Stover, A. G. \& Driks, A. Regulation of synthesis of the Bacillus subtilis transition-phase, spore-associated antibacterial protein TasA. Journal of Bacteriology 181, 5476-5481 (1999).

28 Erskine, E. et al. Formation of functional, non-amyloidogenic fibres by recombinant Bacillus subtilis TasA. Mol Microbiol 110, 897-913, doi:10.1111/mmi.13985 (2018).

29 Diehl, A. et al. Structural changes of TasA in biofilm formation of Bacillus subtilis. Proc Natl Acad Sci U S A 115, 3237-3242, doi:10.1073/pnas.1718102115 (2018).

This study revealed the crystal structure of monomeric TasA and demonstrated that, unlike other biofilm fibre-forming proteins, TasA is folded and globular when monomeric.

30 Azulay, D. N. et al. Colloidal-like aggregation of a functional amyloid protein. Phys Chem Chem Phys 22, 23286-23294, doi:10.1039/d0cp03265d (2020).

31 Steinberg, N. et al. The extracellular matrix protein TasA is a developmental cue that maintains a motile subpopulation within Bacillus subtilis biofilms. Sci Signal 13, doi:10.1126/scisignal.aaw8905 (2020).

32 Candela, T. et al. CalY is a major virulence factor and a biofilm matrix protein. Mol Microbiol 111, 1416-1429, doi:10.1111/mmi.14184 (2019).

33 Arnaouteli, S. et al. Bifunctionality of a biofilm matrix protein controlled by redox state. Proc Natl Acad Sci U S A 114, E6184-E6191, doi:10.1073/pnas.1707687114 (2017).

This study used a multidisciplinary approach that highlighted the genetically distinct roles of the matrix protein BslA.

34 Ostrowski, A., Mehert, A., Prescott, A., Kiley, T. B. \& Stanley-Wall, N. R. YuaB functions synergistically with the exopolysaccharide and TasA amyloid fibers to allow biofilm formation by Bacillus subtilis. J Bacteriol 193, 4821-4831, doi:10.1128/JB.00223-11 (2011).

35 Bromley, K. M. et al. Interfacial self-assembly of a bacterial hydrophobin. Proc Natl Acad Sci U S A 112, 5419-5424, doi:10.1073/pnas.1419016112 (2015).

36 Srinivasan, S. et al. Matrix Production and Sporulation in Bacillus subtilis Biofilms Localize to Propagating Wave Fronts. Biophys J 114, 1490-1498, doi:10.1016/j.bpj.2018.02.002 (2018).

37 Seminara, A. et al. Osmotic spreading of Bacillus subtilis biofilms driven by an extracellular matrix. P Natl Acad Sci USA 109, 1116-1121, doi:10.1073/pnas.1109261108 (2012). 
38 Branda, S. S. et al. Genes involved in formation of structured multicellular communities by Bacillus subtilis. J Bacteriol 186, 3970-3979 (2004).

39 Epstein, A. K., Pokroy, B., Seminara, A. \& Aizenberg, J. Bacterial biofilm shows persistent resistance to liquid wetting and gas penetration. P Natl Acad Sci USA 108, 995-1000, doi:10.1073/pnas.1011033108 (2011).

40 Verhamme, D. T., Murray, E. J. \& Stanley-Wall, N. R. DegU and Spo0A jointly control transcription of two loci required for complex colony development by Bacillus subtilis. $J$ Bacteriol 191, 100-108, doi:10.1128/JB.01236-08 (2009).

41 Kovacs, A. T. \& Kuipers, O. P. Rok Regulates yuaB Expression during Architecturally Complex Colony Development of Bacillus subtilis 168. J Bacteriol 193, 998-1002, doi:JB.01170-10 [pii]

10.1128/JB.01170-10 (2011).

42 Gerwig, J., Kiley, T. B., Gunka, K., Stanley-Wall, N. \& Stulke, J. The protein tyrosine kinases EpsB and PtkA differentially affect biofilm formation in Bacillus subtilis. Microbiology, doi:10.1099/mic.0.074971-0 (2014).

43 Zhu, B. \& Stulke, J. SubtiWiki in 2018: from genes and proteins to functional network annotation of the model organism Bacillus subtilis. Nucleic Acids Res 46, D743-D748, doi:10.1093/nar/gkx908 (2018).

44 Guttenplan, S. B., Blair, K. M. \& Kearns, D. B. The EpsE flagellar clutch is bifunctional and synergizes with EPS biosynthesis to promote Bacillus subtilis biofilm formation. PLoS Genet 6, e1001243, doi:10.1371/journal.pgen.1001243 (2010).

45 Roux, D. et al. Identification of Poly-N-acetylglucosamine as a Major Polysaccharide Component of the Bacillus subtilis Biofilm Matrix. J Biol Chem 290, 19261-19272, doi:10.1074/jbc.M115.648709 (2015).

46 Kaundinya, C. R., Savithri, H. S., Krishnamurthy Rao, K. \& Balaji, P. V. In vitro characterization of N-terminal truncated EpsC from Bacillus subtilis 168, a UDP-N-acetylglucosamine 4,6dehydratase. Archives of biochemistry and biophysics 657, 78-88, doi:10.1016/j.abb.2018.09.005 (2018).

47 Kaundinya, C. R., Savithri, H. S., Rao, K. K. \& Balaji, P. V. EpsN from Bacillus subtilis 168 has UDP-2,6-dideoxy 2-acetamido 4-keto glucose aminotransferase activity in vitro. Glycobiology 28, 802-812, doi:10.1093/glycob/cwy063 (2018).

48 Kaundinya, C. R., Savithri, H. S., Rao, K. K. \& Balaji, P. V. EpsM from Bacillus subtilis 168 has UDP-2,4,6-trideoxy-2-acetamido-4-amino glucose acetyltransferase activity in vitro. Biochem Biophys Res Commun 505, 1057-1062, doi:10.1016/j.bbrc.2018.09.185 (2018).

49 Chai, Y., Beauregard, P. B., Vlamakis, H., Losick, R. \& Kolter, R. Galactose metabolism plays a crucial role in biofilm formation by Bacillus subtilis. MBio 3, e00184-00112, doi:10.1128/mBio.00184-12 (2012).

50 Jones, S. E., Paynich, M. L., Kearns, D. B. \& Knight, K. L. Protection from intestinal inflammation by bacterial exopolysaccharides. J Immunol 192, 4813-4820, doi:10.4049/jimmunol.1303369 (2014).

51 Jakubovics, N. S., Shields, R. C., Rajarajan, N. \& Burgess, J. G. Life after death: the critical role of extracellular DNA in microbial biofilms. Lett Appl Microbiol 57, 467-475, doi:10.1111/lam.12134 (2013).

52 Yu, Y. et al. Poly-gamma-Glutamic Acids Contribute to Biofilm Formation and Plant Root Colonization in Selected Environmental Isolates of Bacillus subtilis. Frontiers in microbiology 7, 1811, doi:10.3389/fmicb.2016.01811 (2016).

53 Ashiuchi, M., Soda, K. \& Misono, H. A poly-gamma-glutamate synthetic system of Bacillus subtilis IFO 3336: gene cloning and biochemical analysis of poly-gamma-glutamate produced by Escherichia coli clone cells. Biochem Biophys Res Commun 263, 6-12 (1999).

54 Kesel, S. et al. Direct Comparison of Physical Properties of Bacillus subtilis NCIB 3610 and B-1 Biofilms. Appl Environ Microbiol 82, 2424-2432, doi:10.1128/aem.03957-15 (2016). 

within microbial biofilms. NPJ Biofilms Microbiomes 2, 15031, doi:10.1038/npjbiofilms.2015.31 (2016).

56 Azulay, D. N. et al. Biopolymers from a Bacterial Extracellular Matrix Affect the Morphology and Structure of Calcium Carbonate Crystals. Cryst Growth Des 18, 5582-5591, doi:10.1021/acs.cgd.8b00888 (2018).

57 Dragos, A. et al. Collapse of genetic division of labour and evolution of autonomy in pellicle biofilms. Nat Microbiol 3, 1451-1460, doi:10.1038/s41564-018-0263-y (2018).

58 Cairns, L. S., Hobley, L. \& Stanley-Wall, N. R. Biofilm formation by Bacillus subtilis: new insights into regulatory strategies and assembly mechanisms. Mol Microbiol 93, 587-598, doi:10.1111/mmi.12697 (2014).

59 Vlamakis, H., Chai, Y., Beauregard, P., Losick, R. \& Kolter, R. Sticking together: building a biofilm the Bacillus subtilis way. Nat Rev Microbiol 11, 157-168, doi:10.1038/nrmicro2960 (2013).

60 Kearns, D. B., Chu, F., Branda, S. S., Kolter, R. \& Losick, R. A master regulator for biofilm formation by Bacillus subtilis. Mol Microbiol 55, 739-749 (2005).

61 Jiang, M., Shao, W., Perego, M. \& Hoch, J. A. Multiple histidine kinases regulate entry into stationary phase and sporulation in Bacillus subtilis. Mol Microbiol 38, 535-543 (2000).

62 McLoon, A. L., Kolodkin-Gal, I., Rubinstein, S. M., Kolter, R. \& Losick, R. Spatial Regulation of Histidine Kinases Governing Biofilm Formation in Bacillus subtilis. J Bacteriol, doi:JB.0118610 [pii]

10.1128/JB.01186-10 (2010).

63 Neiditch, M. B., Capodagli, G. C., Prehna, G. \& Federle, M. J. Genetic and Structural Analyses of RRNPP Intercellular Peptide Signaling of Gram-Positive Bacteria. Annu Rev Genet 51, 311333, doi:10.1146/annurev-genet-120116-023507 (2017).

64 Gallegos-Monterrosa, R. et al. Impact of Rap-Phr system abundance on adaptation of Bacillus subtilis. bioRxiv, doi:10.1101/2020.09.01.278184 (2020).

65 Omer Bendori, S., Pollak, S., Hizi, D. \& Eldar, A. The RapP-PhrP quorum-sensing system of Bacillus subtilis strain NCIB3610 affects biofilm formation through multiple targets, due to an atypical signal-insensitive allele of RapP. J Bacteriol 197, 592-602, doi:10.1128/JB.02382-14 (2015).

66 Banse, A. V., Chastanet, A., Rahn-Lee, L., Hobbs, E. C. \& Losick, R. Parallel pathways of repression and antirepression governing the transition to stationary phase in Bacillus subtilis. Proc Natl Acad Sci U S A 105, 15547-15552 (2008).

67 Milton, M. E. et al. The Solution Structures and Interaction of SinR and Sinl: Elucidating the Mechanism of Action of the Master Regulator Switch for Biofilm Formation in Bacillus subtilis. J Mol Biol 432, 343-357, doi:10.1016/j.jmb.2019.08.019 (2020).

After years of research, this study reports the first solution structures of $\operatorname{SinR}$ and $\operatorname{Sinl}$ and provides experimental data revealing the mechanisms that govern SinR-Sinl interactions.

68 Kobayashi, K. SIrR/SIrA controls the initiation of biofilm formation in Bacillus subtilis. Mol Microbiol 69, 1399-1410 (2008).

69 Chai, Y., Norman, T., Kolter, R. \& Losick, R. An epigenetic switch governing daughter cell separation in Bacillus subtilis. Genes Dev 24, doi:gad.1915010 [pii]

10.1101/gad.1915010 (2010).

70 Chai, Y., Chu, F., Kolter, R. \& Losick, R. Bistability and biofilm formation in Bacillus subtilis. Mol Microbiol 67, 254-263 (2008).

71 Otto, S. B. et al. Privatization of Biofilm Matrix in Structurally Heterogeneous Biofilms. mSystems 5, doi:10.1128/mSystems.00425-20 (2020).

72 Vlamakis, H., Aguilar, C., Losick, R. \& Kolter, R. Control of cell fate by the formation of an architecturally complex bacterial community. Genes Dev 22, 945-953 (2008). 
73 Diethmaier, C. et al. The YmdB phosphodiesterase is a global regulator of late adaptive responses in Bacillus subtilis. J Bacteriol 196, 265-275, doi:10.1128/JB.00826-13 (2014).

74 Diethmaier, C. et al. A novel factor controlling bistability in Bacillus subtilis: the YmdB protein affects flagellin expression and biofilm formation. J Bacteriol 193, 5997-6007, doi:10.1128/JB.05360-11 (2011).

75 Kampf, J. et al. Selective Pressure for Biofilm Formation in Bacillus subtilis: Differential Effect of Mutations in the Master Regulator SinR on Bistability. mBio 9, doi:10.1128/mBio.0146418 (2018).

This study combined genetics and microfluidics to reveal how the interplay between SinR and YmdB determines $B$. subtilis cell fate.

76 Boudvillain, M., Figueroa-Bossi, N. \& Bossi, L. Terminator still moving forward: expanding roles for Rho factor. Curr Opin Microbio/ 16, 118-124, doi:10.1016/j.mib.2012.12.003 (2013).

77 Richardson, J. P. Rho-dependent termination and ATPases in transcript termination. Biochim Biophys Acta 1577, 251-260, doi:10.1016/s0167-4781(02)00456-6 (2002).

78 Bidnenko, V. et al. Termination factor Rho: From the control of pervasive transcription to cell fate determination in Bacillus subtilis. PLoS Genet 13, e1006909, doi:10.1371/journal.pgen.1006909 (2017).

This study revealed that Rho-controlled transcripts constitute a previously unidentified route to control cell differentiation in $B$. subtilis.

79 Oppenheimer-Shaanan, Y., Wexselblatt, E., Katzhendler, J., Yavin, E. \& Ben-Yehuda, S. c-diAMP reports DNA integrity during sporulation in Bacillus subtilis. EMBO Rep 12, 594-601, doi:10.1038/embor.2011.77 (2011).

80 Mehne, F. M. et al. Cyclic di-AMP homeostasis in Bacillus subtilis: both lack and high level accumulation of the nucleotide are detrimental for cell growth. J Biol Chem 288, 2004-2017, doi:10.1074/jbc.M112.395491 (2013).

81 Gundlach, J., Rath, H., Herzberg, C., Mader, U. \& Stulke, J. Second Messenger Signaling in Bacillus subtilis: Accumulation of Cyclic di-AMP Inhibits Biofilm Formation. Frontiers in microbiology 7, 804, doi:10.3389/fmicb.2016.00804 (2016).

82 Townsley, L., Yannarell, S. M., Huynh, T. N., Woodward, J. J. \& Shank, E. A. Cyclic di-AMP Acts as an Extracellular Signal That Impacts Bacillus subtilis Biofilm Formation and Plant Attachment. mBio 9, doi:10.1128/mBio.00341-18 (2018).

This work unravels how cyclic di-AMP affects biofilm development in B. subtilis as a unique intraspecies extracellular signal.

83 Chen, Y., Chai, Y., Guo, J. H. \& Losick, R. Evidence for cyclic Di-GMP-mediated signaling in Bacillus subtilis. Journal of Bacteriology 194, 5080-5090, doi:10.1128/JB.01092-12 (2012).

84 Gao, X. et al. Functional characterization of core components of the Bacillus subtilis cyclic-diGMP signaling pathway. J Bacteriol 195, 4782-4792, doi:10.1128/JB.00373-13 (2013).

85 Carabetta, V. J. et al. A complex of YlbF, YmcA and YaaT regulates sporulation, competence and biofilm formation by accelerating the phosphorylation of SpoOA. Mol Microbiol 88, 283300, doi:10.1111/mmi.12186 (2013).

86 DeLoughery, A., Dengler, V., Chai, Y. R. \& Losick, R. Biofilm formation by Bacillus subtilis requires an endoribonuclease-containing multisubunit complex that controls mRNA levels for the matrix gene repressor SinR. Molecular Microbiology 99, 425-437, doi:10.1111/mmi.13240 (2016).

87 Adusei-Danso, F. et al. Structure-Function Studies of the Bacillus subtilis Ric Proteins Identify the Fe-S Cluster-Ligating Residues and Their Roles in Development and RNA Processing. mBio 10, doi:10.1128/mBio.01841-19 (2019).

88 Dubnau, E. J. et al. A protein complex supports the production of SpoOA-P and plays additional roles for biofilms and the K-state in Bacillus subtilis. Mol Microbiol 101, 606-624, doi:10.1111/mmi.13411 (2016). 
89 Cornforth, D. M. \& Foster, K. R. Competition sensing: the social side of bacterial stress responses. Nat Rev Microbiol 11, 285-293, doi:10.1038/nrmicro2977 (2013).

90 Antelmann, H. et al. Expression of a stress- and starvation-induced dps/pexB-homologous gene is controlled by the alternative sigma factor sigmaB in Bacillus subtilis. J Bacteriol 179, 7251-7256, doi:10.1128/jb.179.23.7251-7256.1997 (1997).

91 Nadezhdin, E., Murphy, N., Dalchau, N., Phillips, A. \& Locke, J. C. W. Stochastic pulsing of gene expression enables the generation of spatial patterns in Bacillus subtilis biofilms. Nat Commun 11, 950, doi:10.1038/s41467-020-14431-9 (2020).

This work demonstrated how stochastic pulsing of gene expression allows for $B$. subtilis cells in different states to co-habit in the same area of the biofilm.

92 Dragos, A. et al. Division of Labor during Biofilm Matrix Production. Curr Biol 28, 1903-1913 e1905, doi:10.1016/j.cub.2018.04.046 (2018).

This study demonstrated a long-standing evolutionary theory of division of labour among $B$. subtilis strains in the production of biofilm matrix components.

93 Andric, S., Meyer, T. \& Ongena, M. Bacillus Responses to Plant-Associated Fungal and Bacterial Communities. Frontiers in microbiology 11, 1350, doi:10.3389/fmicb.2020.01350 (2020).

A review of the secondary metabolites produced by $B$. subtilis species complex in the context of the plant host environment.

94 Yannarell, S. M., Grandchamp, G. M., Chen, S. Y., Daniels, K. E. \& Shank, E. A. A Dual-Species Biofilm with Emergent Mechanical and Protective Properties. J Bacteriol 201, doi:10.1128/JB.00670-18 (2019).

95 Gallegos-Monterrosa, R. et al. LysiniBacillus fusiformis M5 Induces Increased Complexity in Bacillus subtilis 168 Colony Biofilms via Hypoxanthine. J Bacteriol 199, doi:10.1128/JB.0020417 (2017).

96 Duanis-Assaf, D. et al. Cell wall associated protein TasA provides an initial binding component to extracellular polysaccharides in dual-species biofilm. Scientific reports 8,9350 , doi:10.1038/s41598-018-27548-1 (2018).

97 Shank, E. A. et al. Interspecies interactions that result in Bacillus subtilis forming biofilms are mediated mainly by members of its own genus. P Natl Acad Sci USA 108, E1236-1243, doi:10.1073/pnas.1103630108 (2011).

98 Bleich, R., Watrous, J. D., Dorrestein, P. C., Bowers, A. A. \& Shank, E. A. Thiopeptide antibiotics stimulate biofilm formation in Bacillus subtilis. Proc Natl Acad Sci U S A 112, 30863091, doi:10.1073/pnas.1414272112 (2015).

99 Powers, M. J., Sanabria-Valentin, E., Bowers, A. A. \& Shank, E. A. Inhibition of Cell Differentiation in Bacillus subtilis by Pseudomonas protegens. J Bacteriol 197, 2129-2138, doi:10.1128/JB.02535-14 (2015).

100 Miquel Guennoc, C., Rose, C., Labbe, J. \& Deveau, A. Bacterial biofilm formation on the hyphae of ectomycorrhizal fungi: a widespread ability under controls? FEMS Microbiol Ecol 94, doi:10.1093/femsec/fiy093 (2018).

101 Khezri, M., Jouzani, G. S. \& Ahmadzadeh, M. Fusarium culmorum affects expression of biofilm formation key genes in Bacillus subtilis. Brazilian journal of microbiology : [publication of the Brazilian Society for Microbiology] 47, 47-54, doi:10.1016/j.bjm.2015.11.019 (2016).

102 Cawoy, H. et al. Lipopeptides as main ingredients for inhibition of fungal phytopathogens by Bacillus subtilis/amyloliquefaciens. Microb Biotechnol 8, 281-295, doi:10.1111/17517915.12238 (2015).

103 DeFilippi, S., Groulx, E., Megalla, M., Mohamed, R. \& Avis, T. J. Fungal Competitors Affect Production of Antimicrobial Lipopeptides in Bacillus subtilis Strain B9-5. J Chem Ecol 44, 374383, doi:10.1007/s10886-018-0938-0 (2018). 
104 Molina-Santiago, C. et al. The extracellular matrix protects Bacillus subtilis colonies from Pseudomonas invasion and modulates plant co-colonization. Nat Commun 10, 1919, doi:10.1038/s41467-019-09944-x (2019).

105 Muller, S., Strack, S. N., Ryan, S. E., Kearns, D. B. \& Kirby, J. R. Predation by Myxococcus xanthus induces Bacillus subtilis to form spore-filled megastructures. Appl Environ Microbiol 81, 203-210, doi:10.1128/AEM.02448-14 (2015).

106 Prindle, A. et al. Ion channels enable electrical communication in bacterial communities. Nature 527, 59-63, doi:10.1038/nature15709 (2015).

107 Humphries, J. et al. Species-Independent Attraction to Biofilms through Electrical Signaling. Cell 168, 200-209 e212, doi:10.1016/j.cell.2016.12.014 (2017).

108 Arnaouteli, S. et al. Pulcherrimin formation controls growth arrest of the Bacillus subtilis biofilm. Proc Natl Acad Sci U S A 116, 13553-13562, doi:10.1073/pnas.1903982116 (2019).

109 Matoz-Fernandez, D. et al. Comment on "Rivalry in Bacillus subtilis colonies: enemy or family?". Soft Matter 16, 3344-3346, doi:10.1039/c9sm02141h (2020).

110 Ogran, A. et al. The Plant Host Induces Antibiotic Production To Select the Most-Beneficial Colonizers. Appl Environ Microbiol 85, doi:10.1128/AEM.00512-19 (2019).

This study illustrated the complexity $B$. subtilis root colonization and interactions with the plant host, revealing that plants help more beneficial colonizers by inducing their expression of an antibiotic compound.

111 Massalha, H., Korenblum, E., Malitsky, S., Shapiro, O. H. \& Aharoni, A. Live imaging of rootbacteria interactions in a microfluidics setup. Proc Natl Acad Sci U S A 114, 4549-4554, doi:10.1073/pnas.1618584114 (2017).

This is the first report of a microfluidic chip setup to investigate the dynamics of $B$. subtilis root colonization.

112 Rosenberg, G. et al. Not so simple, not so subtle: the interspecies competition between Bacillus simplex and Bacillus subtilis and its impact on the evolution of biofilms. NPJ Biofilms Microbiomes 2, 15027, doi:10.1038/npjbiofilms.2015.27 (2016).

113 Kobayashi, K. \& Ikemoto, Y. Biofilm-associated toxin and extracellular protease cooperatively suppress competitors in Bacillus subtilis biofilms. PLoS Genet 15, e1008232, doi:10.1371/journal.pgen.1008232 (2019).

114 Stefanic, P., Kraigher, B., Lyons, N. A., Kolter, R. \& Mandic-Mulec, I. Kin discrimination between sympatric Bacillus subtilis isolates. Proc Natl Acad Sci U S A 112, 14042-14047, doi:10.1073/pnas.1512671112 (2015).

115 Lyons, N. A. \& Kolter, R. Bacillus subtilis Protects Public Goods by Extending Kin Discrimination to Closely Related Species. MBio 8, doi:10.1128/mBio.00723-17 (2017).

116 Lyons, N. A., Kraigher, B., Stefanic, P., Mandic-Mulec, I. \& Kolter, R. A Combinatorial Kin Discrimination System in Bacillus subtilis. Curr Biol 26, 733-742, doi:10.1016/j.cub.2016.01.032 (2016).

117 Vejan, P., Abdullah, R., Khadiran, T., Ismail, S. \& Nasrulhaq Boyce, A. Role of Plant Growth Promoting Rhizobacteria in Agricultural Sustainability-A Review. Molecules 21, doi:10.3390/molecules21050573 (2016).

118 Blake, C., Christensen, M. N. \& Kovacs, A. T. Molecular Aspects of Plant Growth Promotion and Protection by Bacillus subtilis. Mol Plant Microbe Interact 34, 15-25, doi:10.1094/MPMI08-20-0225-CR (2021).

119 Koua, S. H., N'Golo D, C., Alloue-Boraud, W. M., Konan, F. \& Dje, K. M. Bacillus subtilis Strains Isolated from Cocoa Trees (Theobroma cacao L.) Rhizosphere for their use as Potential Plant Growth Promoting Rhizobacteria in Cote d'Ivoire. Curr Microbiol 77, 2258-2264, doi:10.1007/s00284-020-02027-x (2020).

120 Oslizlo, A. et al. Exploring ComQXPA quorum-sensing diversity and biocontrol potential of Bacillus spp. isolates from tomato rhizoplane. Microb Biotechnol 8, 527-540, doi:10.1111/1751-7915.12258 (2015). 
121 Allard-Massicotte, R. et al. Bacillus subtilis Early Colonization of Arabidopsis thaliana Roots Involves Multiple Chemotaxis Receptors. MBio 7, doi:10.1128/mBio.01664-16 (2016).

This study identified the chemoreceptors that are involved in attraction of $B$. subtilis to host roots, thereby illustrating the active nature of this process.

122 Dong, L. et al. Qualitative and Quantitative Analyses of the Colonization Characteristics of Bacillus subtilis Strain NCD-2 on Cotton Root. Curr Microbiol 77, 1600-1609, doi:10.1007/s00284-020-01971-y (2020).

123 Beauregard, P. B., Chai, Y., Vlamakis, H., Losick, R. \& Kolter, R. Bacillus subtilis biofilm induction by plant polysaccharides. Proc Natl Acad Sci U S A 110, E1621-1630, doi:10.1073/pnas.1218984110 (2013).

This article demonstrated the importance of $B$. subtilis matrix components in plant colonization, and that plant cell wall components induce the production of matrix components by $B$. subtilis.

124 Chen, Y. et al. A Bacillus subtilis sensor kinase involved in triggering biofilm formation on the roots of tomato plants. Mol Microbio/ 85, 418-430, doi:10.1111/j.1365-2958.2012.08109.x (2012).

125 Wang, L., Wang, N., Mi, D., Luo, Y. \& Guo, J. Poly-gamma-glutamic acid productivity of Bacillus subtilis BsE1 has positive function in motility and biocontrol against Fusarium graminearum. J Microbio/ 55, 554-560, doi:10.1007/s12275-017-6589-y (2017).

126 Zhou, H. et al. Loss of GltB Inhibits Biofilm Formation and Biocontrol Efficiency of Bacillus subtilis Bs916 by Altering the Production of gamma-Polyglutamate and Three Lipopeptides. PLoS One 11, e0156247, doi:10.1371/journal.pone.0156247 (2016).

127 Bais, H. P., Fall, R. \& Vivanco, J. M. Biocontrol of Bacillus subtilis against infection of Arabidopsis roots by Pseudomonas syringae is facilitated by biofilm formation and surfactin production. Plant Physiol 134, 307-319 (2004).

128 Therien, M. et al. Surfactin production is not essential for pellicle and root-associated biofilm development of Bacillus subtilis. Biofilm 2, 100021, doi:0.1016/j.bioflm.2020.100021 (2020).

129 Habib, C. et al. Characterization of the regulation of a plant polysaccharide utilization operon and its role in biofilm formation in Bacillus subtilis. PLoS One 12, e0179761, doi:10.1371/journal.pone.0179761 (2017).

130 Hong, H. A. et al. Bacillus subtilis isolated from the human gastrointestinal tract. Res Microbiol 160, 134-143, doi:10.1016/j.resmic.2008.11.002 (2009).

131 Wilson, M. The skin and its indigenous microbiota. In Microbial Inhabitants of Humans: Their Ecology and Role in Health and Disease. (Cambridge University Press, 2004).

132 Jones, S. E. \& Knight, K. L. Bacillus subtilis-mediated protection from Citrobacter rodentiumassociated enteric disease requires espH and functional flagella. Infect Immun 80, 710-719, doi:10.1128/IAI.05843-11 (2012).

133 Paynich, M. L., Jones-Burrage, S. E. \& Knight, K. L. Exopolysaccharide from Bacillus subtilis Induces Anti-Inflammatory M2 Macrophages That Prevent T Cell-Mediated Disease. J Immunol 198, 2689-2698, doi:10.4049/jimmunol.1601641 (2017).

134 Paik, W., Alonzo, F., 3rd \& Knight, K. L. Probiotic Exopolysaccharide Protects against Systemic Staphylococcus aureus Infection, Inducing Dual-Functioning Macrophages That Restrict Bacterial Growth and Limit Inflammation. Infect Immun 87, doi:10.1128/IAI.00791-18 (2019).

135 Garsin, D. A. et al. Long-lived C. elegans daf-2 mutants are resistant to bacterial pathogens. Science 300, 1921, doi:10.1126/science.1080147 (2003).

This is the first experimental data revealing that propagation of $B$. subtilis in $C$. elegans results in extended longevity of the nematode.

136 Donato, V. et al. Bacillus subtilis biofilm extends Caenorhabditis elegans longevity through downregulation of the insulin-like signalling pathway. Nat Commun 8, 14332, doi:10.1038/ncomms14332 (2017). 
This paper demonstrated that biofilm-proficient $B$. subtilis colonizes the $C$. elegans gut and extends nematode lifespan, unlike the biofilm-deficient derivatives.

137 Smolentseva, O. et al. Mechanism of biofilm-mediated stress resistance and lifespan extension in C. elegans. Scientific reports 7, 7137, doi:10.1038/s41598-017-07222-8 (2017).

138 Goya, M. E. et al. Probiotic Bacillus subtilis Protects against alpha-Synuclein Aggregation in C. elegans. Cell Rep 30, 367-380 e367, doi:10.1016/j.celrep.2019.12.078 (2020).

This study links the biofilm-forming capacity of a probiotic $B$. subtilis strain to its protective effect against Parkinson disease in a nematode model.

139 Cogliati, S. et al. Bacillus Subtilis Delays Neurodegeneration and Behavioral Impairment in the Alzheimer's Disease Model Caenorhabditis Elegans. J Alzheimers Dis 73, 1035-1052, doi:10.3233/JAD-190837 (2020).

140 Leiman, S. A., Arboleda, L. C., Spina, J. S. \& McLoon, A. L. SinR is a mutational target for finetuning biofilm formation in laboratory-evolved strains of Bacillus subtilis. BMC Microbiol 14, 301, doi:10.1186/s12866-014-0301-8 (2014).

141 Dragos, A. et al. Evolution of exploitative interactions during diversification in Bacillus subtilis biofilms. FEMS Microbiol Ecol 93, doi:10.1093/femsec/fix155 (2018).

142 Holscher, T. et al. Motility, Chemotaxis and Aerotaxis Contribute to Competitiveness during Bacterial Pellicle Biofilm Development. Journal of molecular biology 427, 3695-3708 (2015).

143 Richter, A. et al. Hampered motility promotes the evolution of wrinkly phenotype in Bacillus subtilis. BMC Evol Biol 18, 155, doi:10.1186/s12862-018-1266-2 (2018).

144 Martin, M. et al. De novo evolved interference competition promotes the spread of biofilm defectors. Nat Commun 8, 15127, doi:10.1038/ncomms15127 (2017).

145 Dragos, A. et al. Pervasive prophage recombination occurs during evolution of spore-forming Bacilli. ISME J, doi:10.1038/s41396-020-00854-1 (2020).

146 Martin, M. et al. Cheaters shape the evolution of phenotypic heterogeneity in Bacillus subtilis biofilms. ISME J 14, 2302-2312, doi:10.1038/s41396-020-0685-4 (2020).

147 Noda-Garcia, L. et al. Chance and pleiotropy dominate genetic diversity in complex bacterial environments. Nat Microbiol 4, 1221-1230, doi:10.1038/s41564-019-0412-y (2019).

148 Bromley, K. M. \& MacPhee, C. E. BsIA-stabilized emulsion droplets with designed microstructure. Interface Focus 7, 20160124, doi:10.1098/rsfs.2016.0124 (2017).

149 Stanley-Wall, N. R. \& MacPhee, C. E. Connecting the dots between bacterial biofilms and ice cream. Phys Biol 12, doi:Artn 063001

10.1088/1478-3975/12/6/063001 (2015).

150 Reeksting, B. J., Hoffmann, T. D., Tan, L., Paine, K. \& Gebhard, S. In-Depth Profiling of Calcite Precipitation by Environmental Bacteria Reveals Fundamental Mechanistic Differences with Relevance to Application. Appl Environ Microbio/ 86, doi:10.1128/AEM.02739-19 (2020).

151 Yahav, S., Berkovich, Z., Ostrov, I., Reifen, R. \& Shemesh, M. Encapsulation of beneficial probiotic bacteria in extracellular matrix from biofilm-forming Bacillus subtilis. Artif Cells Nanomed Biotechnol 46, 974-982, doi:10.1080/21691401.2018.1476373 (2018).

152 Duraj-Thatte, A. M., Dorval Courchesne, N.-M. \& Joshi, N. S. Methods of making gels and films using curli nanofibers. USA patent (2017).

153 Persat, A. et al. The mechanical world of bacteria. Cell 161, 988-997, doi:10.1016/j.cell.2015.05.005 (2015).

154 Werb, M. et al. Surface topology affects wetting behavior of Bacillus subtilis biofilms. NPJ Biofilms Microbiomes 3, 11, doi:10.1038/s41522-017-0018-1 (2017).

155 Falcon Garcia, C. et al. Topographical alterations render bacterial biofilms susceptible to chemical and mechanical stress. Biomater Sci 7, 220-232, doi:10.1039/c8bm00987b (2018).

156 Ruhs, P. A., Boni, L., Fuller, G. G., Inglis, R. F. \& Fischer, P. In-situ quantification of the interfacial rheological response of bacterial biofilms to environmental stimuli. PLoS One 8, e78524, doi:10.1371/journal.pone.0078524 (2013). 
157 Falcon Garcia, C. et al. Metal ions weaken the hydrophobicity and antibiotic resistance of Bacillus subtilis NCIB 3610 biofilms. NPJ Biofilms Microbiomes 6, 1, doi:10.1038/s41522-0190111-8 (2020).

158 Trejo, M. et al. Elasticity and wrinkled morphology of Bacillus subtilis pellicles. Proc Natl Acad Sci U S A 110, 2011-2016, doi:10.1073/pnas.1217178110 (2013).

159 Douarche, C., Allain, J. M. \& Raspaud, E. Bacillus subtilis Bacteria Generate an Internal Mechanical Force within a Biofilm. Biophys J 109, 2195-2202, doi:10.1016/j.bpj.2015.10.004 (2015).

160 Liu, W., Li, S., Wang, Z., Yan, E. C. Y. \& Leblanc, R. M. Characterization of Surface-Active Biofilm Protein BsIA in Self-Assembling Langmuir Monolayer at the Air-Water Interface. Langmuir 33, 7548-7555, doi:10.1021/acs.langmuir.7b01739 (2017).

$161 \mathrm{Ma}, \mathrm{W}$. et al. Bacillus subtilis biofilm development in the presence of soil clay minerals and iron oxides. NPJ Biofilms Microbiomes 3, 4, doi:10.1038/s41522-017-0013-6 (2017).

162. Molecular Microbiology 114 https://onlinelibrary.wiley.com/toc/13652958/2020/114/6 (2020).

Acknowledgements

Á.T.K was supported by the Danish National Research Foundation (DNRF137) for the Center for Microbial Secondary Metabolites (CeMiSt). Work in the laboratory of N.R.S.-W. is funded by the Biotechnology and Biological Science Research Council (BBSRC) (BB/P001335/1 and BB/R012415/1). N.C.B is funded by a long-term fellowship awarded by the European Molecular Biology Organisation (EMBO) (ALTF 471-2020).

Author contributions

The authors contributed equally to all aspects of the article.

Competing interests

The authors declare no competing interests.

Peer review information

Nature Reviews Microbiology thanks Y. Chai, I. Kolodkin-Gal and the other, anonymous, reviewer(s) for their contribution to the peer review of this work.

\section{Display items}

\section{Figure legends}

Figure 1. Formation of Bacillus. subtilis biofilms in vitro. a $\mid$ Schematic of the three modes of biofilm growth in B. subtilis, colony, pellicle and submerged, surface-attached biofilms. b | A 48-hour-old colony biofilm formed by NCIB 3610 ( $90 \%$ founding cells) and an isogenic variant that constitutively expresses green fluorescent protein (GFP; $10 \%$ founding cells) after growth at $30^{\circ} \mathrm{C}$ on an MSgg agar plate. Heterogeneity and patches of clonal derivatives are evident when the biofilm is examined for GFP fluorescence. $\mathbf{c}$ | Confocal microscopy image of the central rugose region of an NCIB 3610 colony biofilm grown for 48 hours on MSgg agar at $30^{\circ} \mathrm{C}$. d | Scanning electron microscopy image of individual cells of B. subtilis 168 in a biofilm. e | NCIB 3610 pellicle biofilm at the air-liquid interface. A water droplet placed on top of the pellicle in the central region (arrow) reveals the hydrophobicity of the biofilm. $\mathbf{f} \mid$ Confocal microscopy image of a submerged biofilm formed by B. subtilis isolate JH642 that is constitutively expressing GFP. Image in part b courtesy of Michael Porter, University of Dundee. Image in part c reprinted with permission from ref. ${ }^{162}$, Wiley. Image in part $\mathbf{d}$ courtesy of Anna Dragoš and Paul J. Kempen, Technical University of Denmark. Image in part f courtesy of Eisha Mhatre, University of Pittsburgh.

Figure 2. Bacillus subtilis biofilm matrix components and biosynthetic systems. a | The biofilm matrix composition is complex and can contain self-produced molecules, including BslA, TasA fibres, extracellular DNA (eDNA), poly- $\gamma$-glutamic acid ( $\gamma$-PGA) and exopolysaccharide (EPS). $\mathbf{b} \mid$ A cartoon schematic of the $\gamma$ PGA (red), EPS (yellow), and TapA (blue/purple) systems showing predicted cell membrane-embedded and cytosolic protein components based on bioinformatics and experimental evidence. The predicted glycosyl transferases (GT) of the EPS system have been labelled based on their GT family membership. SipW (dark blue) post-translationally cleaves TapA (light blue) and TasA (purple) at the cell surface with the arrows representing the location of cleavage. $\mathbf{c}$ | Transmission electron microscopy image of a recombinant BslA film 
with the inset showing the Fast Fourier Transform supporting high order of the lattice. $\mathbf{d} \mid$ Cartoon representation of two monomers from the BslA crystal structure (Protein Data Bank (PDB) identifier (ID): 4BHU) ${ }^{12}$, showing the 'cap in' and the 'cap out' conformation ${ }^{35}$. e | Recombinant TasA forms fibres in vitro that can be visualized by transmission electron microscopy. $\mathbf{f} \mid$ Cartoon representation of the crystal structure of TasA (PDB ID: 5OF1) with $\beta$-strands in purple and $\alpha$-helices in yellow. Part c adapted with permission from ref. ${ }^{35}$, National Academy of Sciences.

Figure 3. Formation of Bacillus subtilis biofilms in the rhizosphere. a $\mid$ Schematic showing the rhizosphere (orange; the area around the growing plant root) with $B$. subtilis biofilms (teal) growing in the elongation zone. These biofilms influence plant growth and behaviour (green arrow) and directly protect against microbial plant pathogens through the section of active compounds. b | Magnified view of plane root cells, showing the three steps of $B$. subtilis colonization, namely attraction (step 1), attachment (step 2) and biofilm formation (step 3). Signals such as plant exudates and bacterially produced cyclic di-adenylate monophosphate (c-di-AMP) are important for attraction and attachment. c| Magnified view of bacterial cells, showing the molecular details of each step of plant root colonization. The chemoreceptors McpB, McpC, TlpC and others (pink) sense plant signals during attraction (step 1). The c-di-AMP permeases YcnB and YhcA (orange) are involved in intercellular signalling during attachment (step 2). The kinases KinC and KinD (green) sense plant polysaccharides and plant produced L-malic acid, which leads to matrix production. Binding of plant cell wall polysaccharides (green hexagon) to an unknown receptor also leads to upregulation of galactan (gan operon), which can lead to EPS production. Biofilm formation (step 3) occurs with the digestion of galactan and the production of matrix components.

Figure 4. Effects of Bacillus subtilis biofilms in the intestinal tract. a | Treatment of mice with the enteric pathogen Citrobacter rodentium (green) causes acute colitis (part aA). Pre-treatment of mice with B. subtilis NCIB 3610 spores (red) prior to inoculation with $C$. rodentium protects against acute colitis (part aB). Pretreatment of mice with $B$. subtilis NCIB 3610 purified EPS (oval dots) and subsequently with C. rodentium also protects against acute colitis (part aC). Model of EPS modulation of immune responses. B. subtilis and purified EPS induce differentiation of M2 macrophages, which inhibit CD4 ${ }^{+}$and $\mathrm{CD}^{+} \mathrm{T}$ cells through the production of TGF $\beta$ and PDL1 (part aD). b | Inhibition of $\alpha$-synuclein aggregation (green triangles) by $B$. subtilis biofilms in a Caenorhabditis elegans synucleinopathy model. Using E. coli strain OP50 (blue) as a food source leads to accumulation of $\alpha$-synuclein aggregates (part bA), whereas using $B$. subtilis NCIB 3610 (red) as a food source results in reduced formation of $\alpha$-synuclein aggregates (part bB). Using B. subtilis NCIB $3610 \Delta \operatorname{tas} A$ (yellow) as a food source removes the protective effect of $B$. subtilis biofilm formation on $\alpha$-synuclein aggregation (part $\mathbf{b C}$ ).

Figure 5. Evolution of social interactions in Bacillus subtilis biofilms. a | Experimental evolution of B. subtilis biofilms revealed genetic differentiation discernible by the appearance of versatile colony morphotypes with altered genetic background. $\mathbf{b}$ | Genetic division of labour leads to increased biofilm productivity compared with transcriptional division of labour. $\mathbf{c} \mid$ The presence of cheaters alters the intrinsic phenotypic heterogeneity during experimental evolution. d| An increase in cheater frequency ultimately leads to the tragedy of the commons (depletion of a shared resource through unhampered use driven by self-interest).

\section{Boxes}

\section{BOX 1. Biophysical techniques in Bacillus subtilis biofilm research}

From a biophysics perspective, biofilms are a form of soft matter - a material that can easily be deformed by external forces and behaves differently to solid and liquids. Biophysical techniques have been used to probe biofilm properties at both the whole biofilm and macromolecular level, yielding novel insights into their form and properties (comprehensively reviewed elsewhere ${ }^{153}$ ).

\section{[b1] Sessile drop methods}

These methods are used for direct measurement of the contact angle between a liquid and a surface and revealed that the upper surface of the Bacillus subtilis colony biofilm is non- 
wetting $^{39}$, a finding that led to molecular analyses of the mechanisms underpinning this remarkable feature $12,13,33,35,141,154,155$.

\section{[b1] Pendant drop analysis}

This type of analysis allows the measurement of surface and interfacial tension and was used to show that BslA is a surface-active protein that forms a stable film at an interface ${ }^{12}$.

\section{[b1] Rheology}

Rheology is the study of how soft matter deforms and flows. Rheological analysis in the presence and absence of specific matrix elements has revealed that biofilms are viscoelastic structures with properties that change over time ${ }^{156}$ or in evolved strains ${ }^{57}$ and dual-species biofilms $^{94}$ and that the BslA surface layer adds stiffness to the biomass ${ }^{54}$.

\section{[b1] Profilometric imaging}

This technique has been used to analyse surface topology at the macro- and meso-scale, revealing how the biofilm surface complexity affects wetting behaviour ${ }^{154}$ and susceptibility to chemical and mechanical stresses ${ }^{155}$, the effect of metal ions on biofilm surface wetting ${ }^{157}$, and the disassembly of experimentally evolved colony morphotypes.

\section{[b1] Langmuir trough}

This apparatus allows the effect of a compressive force on molecules (across a range of scales) on the surface of a subphase to be determined. Studies with the Langmuir trough revealed that the elastic properties of the whole pellicle biofilm are conferred by the extracellular matrix and that the wrinkled morphology is derived from compression by growth in a confined space ${ }^{158}$, 159. On a smaller scale, the properties of the stable film of BslA assembled at the air-liquid interface have been probed ${ }^{160}$.

\section{[b1] Atomic force microscopy}

A form of scanning probe microscopy that has been used to examine B. subtilis biofilms on clay particles ${ }^{161}$ and to investigate the interaction between extracellular DNA and exopolysaccharide to probe the space between cells and uncover detail of the microstructure of the biofilm surface ${ }^{14}$. 


\section{BOX 2. Imaging techniques to study plant colonization}

Plant-microbe interactions are complex and challenging to investigate at high resolution in real time. Root colonization and biofilm formation in the rhizosphere have been studied primarily using endpoint assays in which seedlings or plants are exhumed from the growth media or soil. Advances in microfluidic setups have enabled the visualization of biofilm formation on the roots of thale cress (Arabidopsis thaliana), cotton plants and aspen trees. These studies customized microfluidic chip-based plant cultivation experiments (see the figure) to investigate root-microbe interactions (RMIs) over time. RMI chip systems have been designed to contain an array of isolated growth chambers with individual media inlets and outlets, allowing for a comparison of conditions and replicates in the same microfluidic device ${ }^{8,111}$. Colonization and biofilm dynamics at single-cell resolution can be investigated with RMI chip systems using confocal microscopy, such as investigating Bacillus subtilis preference between different $A$. thaliana genotypes and the interplay between B. subtilis biofilms and Escherichia coli at the root surface ${ }^{111}$. These experiments are somewhat limited by the small chamber size required for microfluidics, as fast-growing plants such as A. thaliana out-grow the chamber in about 10 days, whereas slower-growing plants such as tree seedlings could be visualized for much longer ${ }^{8}$.

\section{ToC blurb}

In this Review, Stanley-Wall and colleagues provide an overview of biofilm composition and formation in Bacillus subtilis and how this research is informing microbial evolution and ecology and aiding in the development of beneficial applications for biofilms. 